\title{
Motility Control Through an Anti-Activation Mechanism
}

\author{
in Agrobacterium tumefaciens
}

\author{
Melene A. Alakavuklar, Brynn C. Heckel, Ari M. Stoner, Joseph A. Stembel, \\ and Clay Fuqua \\ Indiana University, Bloomington, Indiana
}

Address for correspondence: Clay Fuqua, Dept. Biol., 1001 E. 3rd St., Jordan Hall 142, Indiana Univ., Bloomington, IN 47405-1847. Tel: 812-856-6005, FAX: 812-855-6705, Email: cfuqua@indiana.edu

Current addresses: BCH, California State University, Dominguez Hills, CA; AMS, Indiana University Medical School; JAS, University of Washington, Seattle 


\section{Summary}

Many bacteria can migrate from a free-living, planktonic state to an attached, biofilm existence. One factor regulating this transition in the facultative plant pathogen Agrobacterium tumefaciens is the ExoR-ChvG-Chvl system. Periplasmic ExoR regulates activity of the ChvG-Chvl two-component system in response to environmental stress, most notably low $\mathrm{pH}$. Chvl impacts hundreds of genes, including those required for type VI secretion, virulence, biofilm formation, and flagellar motility. Previous studies revealed that activated ChvG-Chvl represses expression of most of class II and class III flagellar biogenesis genes, but not the master motility regulators vis $N$, visR, and rem. In this study, we characterized the integration of the ExoR-ChvGChvl and VisNR-Rem pathways. We isolated motile suppressors of the non-motile $\triangle e x o R$ mutant and thereby identified the previously unannotated mirA gene encoding a 76 amino acid protein. We report that the MirA protein interacts directly with the Rem DNA-binding domain, sequestering Rem and preventing motility gene activation. The ChvG-Chvl pathway activates mirA expression and elevated mirA is sufficient to block motility. This study reveals how the ExoR-ChvG-Chvl pathway prevents flagellar motility in A. tumefaciens. MirA is also conserved among other members of the Rhizobiales suggesting similar mechanisms of motility regulation.

\section{Plain language summary}

Agrobacterium tumefaciens is a plant pathogen that responds to low $\mathrm{pH}$, stressful environments via a complex two-component regulatory system. This regulatory system 
bioRxiv preprint doi: https://doi.org/10.1101/2021.06.24.449765; this version posted June 24, 2021. The copyright holder for this preprint (which was not certified by peer review) is the author/funder, who has granted bioRxiv a license to display the preprint in perpetuity. It is made available under aCC-BY-NC 4.0 International license.

24 can completely inhibit bacterial locomotion by flagella, and in this study we define the

25 mechanism for this inhibition and identify a novel small protein regulator that blocks

26 flagellar gene expression.

27 


\section{Introduction}

The bacterial flagellum is one of the most complex and sophisticated externalized structures that bacteria produce. Flagellar biosynthesis genes in bacteria are under hierarchical regulation, resulting in morphogenetic control of flagellum assembly (Chevance \& Hughes, 2008, Soutourina \& Bertin, 2003). The proper temporal and stoichiometric control of flagellar biogenesis dictates the inside-out assembly of flagella and provides checkpoints to ensure that the requisite flagellar components are produced in sufficient quantities as they are required (Chevance \& Hughes, 2017). Based on the best-studied systems in Escherichia coli and Salmonella enterica as models, the top genes of the hierarchy (class I) encode transcription factors that activate expression of genes specifying the flagellar machinery components (class II) (Kawagishi et al., 1992). Class II genes encode proteins that make up the hook-basal body complex of the flagellum. The basal body includes the type III secretion system that exports hook and filament proteins outside of the cell for filament assembly and the torque-generating motor proteins required for filament rotation. Assembly of the basal body permits class II proteins to activate expression of class III genes (Chevance \& Hughes, 2008). In the Gammaproteobacteria (such as E. coli, S. enterica, and Pseudomonas aeruginosa) and the Gram-positive bacterium Bacillus subtilis, class II genes encode the entire hook-basal body complex, and class III genes include hook-

47 filament junction proteins and flagellin proteins (Mukherjee \& Kearns, 2014). 
51 consists of the LuxR-FixJ-type transcription factors VisN and VisR (Rotter et al., 2006,

52 Sourjik et al., 2000, Tambalo et al., 2010, Xu et al., 2013). When either visN

(ATU_RS02580) or visR (ATU_RS02585) are absent, transcription of flagellar biosynthesis and chemotaxis genes is abolished and cells are non-motile. Vis N and VisR are predicted to function as heteromultimers because of the redundancy of their single mutant phenotypes (Sourjik et al., 2000). In Sinorhizobium meliloti, the motility gene expression defect of visNR mutants is directly caused by loss of expression of the class IB gene, rem (Rotter et al., 2006). Rem is an orphan OmpR-like transcription factor that is required for expression of all motility and chemotaxis genes. Rem from $S$. meliloti directly binds to and activates expression of several promoters that control

61 flagellar basal body protein-encoding genes (Rotter et al., 2006). The hierarchical system of motility gene transcription in APB requires the class IB gene rem for expression of all class II and III flagellar and chemotaxis genes. Ectopic expression of rem in class IA visNR mutants is epistatic to the $\Delta$ vis $N R$ motility defect, driving normal motility and demonstrating the regulatory hierarchy in this pathway. are repressed in acidic conditions via the pH-responsive ExoR-ChvG-Chvl signaling system (Heckel et al., 2014, Yao et al., 2004, Yuan et al., 2008). In neutral conditions, the periplasmic protein ExoR binds to the sensor kinase ChvG and prevents initiation of phosphotransfer to Chvl in this two-component system (Chen et al., 2008, Wells et al.,

71 2007). Upon cultivation in low pH growth media, ExoR is proteolytically inactivated,

72 unfettering the ChvGl system, and impacting a large regulon (Lu et al., 2012). In $A$.

73 tumefaciens, the activity of ChvG-Chvl leads to dramatic decreases in motility gene 
expression (Heckel et al., 2014). The expression of nearly every motility-related gene is decreased when $A$. tumefaciens cells encounter acidic conditions, including both flagellar biosynthetic gene clusters and chemotaxis genes found throughout the $A$. tumefaciens genome. This phenomenon occurs whether cells are exposed to low $\mathrm{pH}$ media or in an $\Delta$ exoR (ATU_RS08400) mutant (Heckel et al., 2014, Yuan et al., 2008). The mechanism by which activated ChvG-Chvl so effectively represses motility gene expression has remained unclear. We hypothesize that the low pH-activated ChvG-Chvl system functions to disrupt the flagellar gene expression hierarchy.

Another complex phenotype that is regulated by environmental $\mathrm{pH}$ in $\mathrm{A}$. tumefaciens is attachment to surfaces. The acid-responsive ExoR-ChvG-Chvl system has a dramatic effect on attachment and biofilm formation: $\Delta$ exoR mutants are completely deficient for attachment and fail to form biofilms on both abiotic and plant surfaces (Tomlinson et al., 2010). A. tumefaciens cells grown in acidic conditions also fail to form biofilms. The attachment defect of acid-exposed cells and $\Delta e x O R$ mutants is dependent on the two-component system ChvG-Chvl, and mutation of either regulator gene in an $\Delta$ exoR mutant background restores biofilm formation capabilities (Heckel et al., 2014). Thus, an active ChvG-Chvl system serves to inhibit both swimming motility and biofilm formation. The uniformly negative impact that active ChvG-Chvl has on motility and attachment is atypical, because these two complex phenotypes are often considered as inverse processes; that is, bacteria will progress through either a motile or sessile program depending on the chemical profile of their environment (Prüß, 2017). The VisN and VisR regulators have a reciprocal impact on motility and biofilm formation (Xu et al., 2013). visN and visR mutants are non-motile but also over-produce the 
97 adhesive unipolar polysaccharide (UPP), which results in a hyper-attachment phenotype. This is despite the observation that non-motile and aflagellate $A$. tumefaciens mutants have a significant deficiency in surface attachment under nonflowing conditions, a phenotype likely due to inefficient engagement with surfaces (Merritt et al., 2007). The increased UPP production in visN and visR mutants is due to elevated levels of the intracellular second messenger cyclic diguanylate monophosphate (cdGMP) and is mediated through control of specific cyclic-di-GMP synthases (diguanylate cyclases, DGCs) (Xu et al., 2013). It is unclear to what extent the ExoR regulon intersects with the VisNR regulon to coordinate the complex developmental phenotypes of motility and biofilm formation. We previously described transcriptome analyses for both the VisNR regulon (Xu et al., 2013) and the ExoR regulon (Heckel et al., 2014). The VisNR regulon was determined by comparing the transcriptomes of a $\Delta v i s R$ strain and wild-type $A$. tumefaciens, and the ExoR regulon was determined by comparing $\Delta e x o R$ to wild-type.

111 The VisNR and ExoR regulons shared a striking congruence of down-regulated genes

112 in the motility and chemotaxis category; 51 genes from over a dozen operons display severely reduced expression in both analyses. This is not surprising for the VisNR

114 regulatory network; VisNR are class IA motility regulator proteins, so we expect expression of all flagellar synthesis and chemotaxis genes to be dependent on Vis $\mathrm{N}$ and VisR. Importantly, expression of rem (ATU_RS02820), encoding the class IB regulator was greatly reduced in the $\Delta v i s R$ mutant compared to wild-type. Although almost all the motility and chemotaxis genes are also dramatically decreased in the $\Delta$ exoR mutant, 119 conspicuously absent are the class I motility regulators vis $N$, visR, and rem (Heckel et 
al., 2014). These findings suggest that the ExoR-ChvG-Chvl pathway impacts motility downstream of rem expression. In this study, we examined the interaction between these pathways and discovered a previously undefined ExoR-ChvG-Chvl-regulated target gene that directly impacts Rem-dependent gene activation.

\section{Results}

\section{Convergence of the VisN-VisR-Rem and ExoR-ChvG-Chvl Pathways}

We previously demonstrated that deletion of the chvl gene (ATU_RS00165) relieves the inhibition of motility and chemotaxis genes in the $\triangle$ exoR (ATU_RS08400) mutant (Heckel et al., 2014). To further investigate the role of chvl, we examined the impact of mutating its site of phosphorylation. As with many two-component system response regulators, Chvl is predicted to be phosphorylated at a conserved aspartate residue (D52) in its $\mathrm{N}$-terminal receiver domain to become active, in this case via the activity of the ChvG sensor kinase (Fig. S1) (Cheng \& Walker, 1998). We mutated the chvl codon for this aspartate to glutamate (D52E) and to asparagine (D52N), to mimic and prevent phosphorylation by ChvG, respectively. Allelic replacement mutants of the native chvl gene with these alleles in the wild type and the $\Delta$ exoR mutant were then tested for control of motility and biofilm formation. Similar to $\triangle e x o R$, the chvID52E mutant was non-motile and had a severe biofilm defect; the phenotypes of this mutant were not impacted by deletion of exoR (Fig. 1A). In contrast, the chvID52N mutation had no effect on motility in an otherwise wild-type background but was completely epistatic to the non-motile and non-biofilm $\triangle$ exoR mutant phenotypes. The chvID52E mutant also phenocopies the $\Delta$ exoR deletion strain for elevated succinoglycan 
143 production (Heckel et al., 2014, Tomlinson et al., 2010), evidenced by dramatic binding

144 of Calcofluor, a polysaccharide-specific dye, and diffuse fluorescence around the colony

145 (Fig. S2). In contrast, when the native copy of chvl was replaced with the chvID52N

146 allele, colonies show a slight decrease in Calcofluor binding relative to wild type (Fig

147 S2). The $\triangle \operatorname{exoR} \triangle \operatorname{exo}($ ATU_RS18935) double mutant does not produce

148 succinoglycan, and does not stain with Calcofluor (Tomlinson, 2010 \#1702).

150 translational fusion (Fig. 1B) nor does it impact a similar lacZ translational fusion to visN

151 (Fig. S3). These results are consistent with transcriptomic analysis of the $\Delta$ exoR mutant,

152 as there is no substantial reduction of vis $N$, visR, or rem transcript abundance in this

153 genetic background (Heckel et al., 2014). In fact, rem-lacZ expression remains

154 unchanged in the $\Delta c h v l$, chvID52E, and chvID52N mutants (Fig. 1B). In contrast,

155 similarly constructed lacZ fusions to the motility genes motA (ATU_RS02760) and flgE

156 (ATU_RS02825), encoding a motor protein and the flagellar hook protein respectively,

157 were greatly diminished in the $\triangle e x O R$ and chvID52E mutants, but equivalent to wild type

158 in the $\Delta c h v l$ null and chvID52N mutants. Conversely, activity of lacZ fusions to chvl itself

159 and the predicted outer membrane protein aopB (ATU_RS05585) were strongly

160 elevated in $\triangle e x O R$ and chvID52E mutants (Fig. 1B); these results correlate well with

161 prior transcriptomic data suggesting that the transcription of $c h v l$ and aopB are elevated

162 in the $\Delta$ exoR mutant (Heckel et al., 2014). The aopB fusion was decreased relative to wild type in $\Delta c h v l$ and chvID52N mutants, whereas the chvl-lacZ was equivalent to wild

164 type. Thus, although these data suggest that phospho-Chvl activates expression of 165 target genes such as aop $B$, it inhibits motility gene expression, and does so 
166 independent of changes to rem or visNR expression. Therefore, we explored other

167 models of how Chvl might be regulating motility gene expression.

Chvl and Rem independently bind their own target promoters but do not cross-

171 model for Chvl-mediated inhibition of flagellar motility gene expression was that Chvl

172 directly competes with Rem for binding to Rem-dependent motility promoters thereby

173 preventing their transcriptional activation. Chvl is a PhoB/OmpR class response

regulator, with a winged helix-turn-helix motif, and it regulates many genes in $A$. operons that initiate with impA (ATU_RS20340) and clpV (ATU_RS20345) (Wu et al., derivatives of $\mathrm{Chvl}^{\mathrm{D} 52 \mathrm{E}}$ and wild type $\mathrm{Chvl}$ to bind to a PCR amplicon of this promoter region (defined as $P_{h c p}$ ) in vitro as determined by electrophoretic mobility shift assay (EMSA) (Fig. 2A-B). Consistent with the prior study, His6-Chvl ${ }^{\mathrm{D} 52 \mathrm{E}}$ was able to robustly shift the $P_{\text {hcp }}$ DNA fragment (Fig. 2A). In contrast to the prior study, however, we observed similar binding of $P_{h c p}$ by the wild-type His6-Chvl allele, presumably the nonphosphorylated form of the protein (Fig. 2B). However, addition of either protein does not shift the mobility of DNA fragments from any of the upstream regions of motility genes (data not shown). To determine more specifically where Chvl binds the hcp 
189 that at least $140 \mathrm{bp}$ upstream of the start codon of $c / p V$ were required in the amplicon to observe a gel mobility shift following incubation with His6-Chvl ${ }^{\mathrm{D} 52 \mathrm{E}}$, well beyond the transcription start site we mapped by 5' RACE to be 63 bp upstream of the start codon

192 of clpV. We also used 5' RACE to map the transcription start sites of chvl and aopB, and found them to start 84 and 142 bp upstream of the start codon, respectively.

194 However, we did not observe His6-Chvl ${ }^{\mathrm{D} 52 \mathrm{E}}$ binding to these promoters by EMSA (data not shown).

Rem is a two-component-type response regulator, with no known cognate sensor kinase, and it initially seemed plausible that ChvG might act to phosphorylate Rem as well as Chvl (although that model would not readily explain the impact of the chvID52E mutant on motility gene expression). Alignment of Rem with well-characterized response regulators indicates that, at the position for the conserved aspartic acid that is

201 the phosphorylation site of canonical response regulators, Rem instead has a glutamate 202 residue at this position (E50; Fig. S1). However, there is an aspartate five residues more N-terminal to this site (D45). To test whether these residues are important for Rem 204 function, we expressed rem with the glutamate (RemE50N) or with the upstream aspartate residue (RemD45N), mutated to an asparagine. Expression of the RemE50N

206 allele complemented a $\Delta$ rem mutant for motility (Fig S5A) and biofilm formation (Fig 207 S5B) similarly to the wild-type rem allele and had no impact on the non-motile exoR 208 phenotype. The RemD45N allele partially restored motility and biofilm formation to the $\Delta$ rem mutant. Together, these results suggest that the native residues D45 and E50 are not required for the ability of Rem to bind the class II motility promoters, although the

211 D45N mutation may partially compromise Rem activity. 
The Rem protein belongs to the emerging family of aspartate-less receiver domain (ALR)-containing response regulators, which have receiver domains lacking the canonical aspartate residue that is typically the site of phosphorylation leading to

215 modulation of the protein's activity (Maule et al., 2015). A subset of this protein family 216 contain a single cysteine residue in this or nearby position. One such ALR-containing 217 protein, RitR from Streptococcus pneumoniae, contains a single cysteine residue in the 218 linker region between the receiver and DNA-binding domain and requires the cysteine 219 residue for redox state-dependent dimerization, thereby impacting gene regulation 220 (Glanville et al., 2018). Mutation of the cysteine to serine or aspartate simulates a 221 reduced or oxidized state, respectively and modulates DNA-binding activity. Rem contains a single cysteine at position 143 and we hypothesized that Rem might function similarly (Fig. S1). However, expression of rem alleles with Rem143C mutated to serine, 224 aspartate or alanine complemented motility equivalently to the wild-type allele (Fig. S6).

225 The cysteine residue in this position is also not conserved in Rem orthologs (Fig. S1). The transcriptional start sites of Rem-regulated class II flagellar genes flgB (ATU_RS02735) and motA (ATU_RS02760) were determined by 5' RACE mapping (Rapid Amplification of cDNA ends) and found to be downstream of an imperfect direct

229 repeat sequence that is conserved among multiple $A$. tumefaciens predicted class II motility gene promoters (CG-WCAAGWCTCRCG-CAAGNYYNNAC; Fig. S7A) and

231 similar to repeats bound by Rem upstream of motility genes in S. meliloti (Rotter, 2006 232 \#1465). The divergent motility gene operons that initiate with ATU_RS02755 and motA 233 have two of these direct repeat sequences in their intergenic region. Rem was purified using an Intein tag system (in which the tag self processes to release a nearly native 
235 protein). When complexed with PCR-amplified $P_{\text {motA, }}$ Rem forms two shifted EMSA complexes (Fig. 2C), indicative of two Rem binding sites. The $P_{f l g B}$ fragment only contains a single putative Rem binding site and forms a single Rem-DNA complex (Fig. S7B), which suggests that Rem binds one site at this promoter. We did not observe binding of Rem to $P_{\text {rem }}$ (Fig. S7C). To test whether Chvl competes with Rem for DNA binding, we incubated purified His6-Chvl ${ }^{\mathrm{D} 52 \mathrm{E}}$ with Rem and the $P_{\operatorname{mot}}$ amplicon. We assessed binding by mixing all three components (Rem, His6-Chvl ${ }^{\mathrm{D} 52 \mathrm{E}}$, and $P_{\text {motA }}$ )

242 simultaneously and also with addition of His6-Chvl ${ }^{\mathrm{D} 52 \mathrm{E}}$ to pre-formed Rem-DNA complexes. We did not observe specific binding of His6-Chvl ${ }^{\mathrm{D} 52 \mathrm{E}}$ to $P_{\operatorname{mot}}$, nor did His6Chvl ${ }^{\mathrm{D} 52 \mathrm{E}}$ disrupt Rem- $P_{\text {motA }}$ complexes (Fig. 2D, S8A) other than at very high protein concentrations that led to non-specific, large aggregates.

247 Chvl might directly interact with Rem. To test for interaction between these proteins, we performed farwestern assays, immobilizing Rem on nitrocellulose membranes and probing with $\mathrm{His}_{6}-\mathrm{Chvl}^{\mathrm{D} 52 \mathrm{E}}$. Upon development with monoclonal anti-His6 antibody, we did not observe stable association of Chvl and Rem (Fig. S8B). Overall, the genetic interaction we have observed between the ExoR-ChvG-Chvl and the VisNR-Rem

252 pathways could not be explained by physical interaction between Chvl and Rem or Chvl 253 and the target promoters of Rem. We therefore hypothesized that a previously254 unidentified Chvl-regulated factor inhibits Rem-dependent motility gene activation upon 255 growth in acidic medium or in upon deletion of exoR.

256 Isolation of motile suppressors of $\Delta$ exoR 
To identify a cellular component that mediates the motility inhibition by Chvl, we performed a transposon-mediated motility suppressor screen to identify mutants that restore motility to a $\triangle e x o R \triangle e x o A$ double mutant (exoA is mutated to prevent the mucoidy associated with succinoglycan overproduction in the $\Delta e x o R$ mutant) (Heckel et al., 2014). For simplicity, we will list the $\triangle \operatorname{exo} R \triangle e x o A$ double mutant strain as $\Delta e x o R$ unless otherwise specified. As observed before and therefore expected, multiple transposon insertional mutations were identified in the chvl-chvG locus. In addition to reversing motility, these null mutations also rescue the biofilm deficiency of $\Delta$ exoR (Heckel et al., 2014). Expression of the chvID52N allele fails to correct the biofilm

266 formation of $\Delta \operatorname{exoR}$ (Fig. 1A), indicating that the biofilm deficiency of $\Delta e x o R$ is 267 dependent on the phosphorylation of Chvl. Although flagellar motility is correlated with efficient biofilm formation in A. tumefaciens (Merritt et al., 2007), the biofilm deficiency of the $\Delta$ exoR mutant is more severe than that for the non-motile mutants such as $\Delta r e m$,

270 indicating that there are additional functions besides motility under ExoR control that 271 impact biofilm formation (Fig. S9). We screened for $\Delta$ exoR suppressor mutants that

272 rescued the motility defect but retained the biofilm deficiency of $\Delta e x o R$, and therefore 273 retained native chvG-chvl. We obtained three such motile, biofilm-deficient transposon 274 mutants $\mathrm{BCH} 132, \mathrm{BCH} 133$, and $\mathrm{BCH} 134$ (Fig. 3A, Fig. S10A). To confirm that the 275 suppressor mutations do not alter the activity of ChvG-Chvl, we assessed expression of 276 the Chvl-regulated genes $c h v l$ and $a o p B$ through activity of lacZ translational fusions in

$277 \mathrm{BCH} 133$ (Fig. S10B). We found that $\mathrm{BCH} 133$ retained the elevated $P_{c h v l}$ and $P_{\text {aopB }}$ 278 activity indicative of active of ChvG-Chvl. Since $\Delta$ exoR has a nonmotile phenotype but 279 does not alter rem expression (Heckel et al., 2014), we hypothesized that these 
suppressors of $\Delta$ exoR would restore motility without changing rem expression. Indeed, we observed no change in $P_{\text {rem }}$ activity in $\mathrm{BCH} 133$ relative to its $\triangle$ exoR $\triangle$ exoA parent strain (Fig. S10B). Sequencing at the transposon junctions revealed $\mathrm{BCH} 132 \mathrm{had}$ a transposon insertion within ATU_RS03690, a bioY homolog likely involved in biotin biosynthesis. The transposon in BCH133 mapped to ATU_RS10935, which is predicted to code for an $\mathrm{ABC}$ transporter subunit. $\mathrm{BCH} 134$ carried an insertion within ATU_RS09055, which is homologous to bolA from E. coli, which negatively regulates motility and positively regulates biofilm formation (Dressaire et al., 2015). Although we hypothesized that BolA may regulate these phenotypes in A. tumefaciens, deletion of bolA in the $\triangle$ exoR mutant did not restore motility or biofilm formation, nor did the deletion independently affect these phenotypes (Fig. S11A-B). Since all three transposon mutants had similar phenotypes with unrelated transposon insertion sites, we subjected $\mathrm{BCH} 132-4$ to whole-genome resequencing. The single mutations from all three isolates clustered to the same general genomic location, diagrammed in Fig $3 \mathrm{~A}$ and were first mapped to the initial $A$. tumefaciens C58 reference genome. The independent mutations included a single base substitution (BCH134), a base deletion in the 3' end of the annotated hypothetical gene Atu1638 (BCH133), and a single base substitution in the intergenic region between Atu1638 and the adjacent gene Atu8018 (ATU_RS08045) (BCH132). We recreated the mirA frameshift suppressor mutation of $\mathrm{BCH} 133$ in a naïve $\Delta \mathrm{exO} R$ background $\left(\Delta \mathrm{exo} R\right.$ mirA $\left.A_{\mathrm{Fs}}\right)$; the mutation restored motility to $\Delta e x o R$ (Fig. S10C) but retained the biofilm defect of the parent strain (data not shown). The point mutation had no effect on swimming motility in the wild-type background, suggesting that motility may be saturated at wild type levels, or that the effects of this 
303 mutation are specific to an $\Delta e x o R$ background. Given the proximity of the mutations and 304 the fact that they all shared the same phenotype, the locus was investigated further. Introduction of an in-frame stop codon after amino acid 19 (serine) within the Atu1638 coding sequence did not restore motility to $\Delta$ exoR (Fig. S12A), suggesting that Atu1638 may not be required for the motility defect of $\Delta$ exoR. A survey for over-annotated genes performed by Yu et al. predicted that Atu1638 was erroneously annotated as a proteincoding gene (Yu et al., 2015), and Atu1638 is not annotated in a more recent C58 genome annotation.

$312 \mathrm{bp})$ overlaps all three mutations; this reading frame was not annotated in the $A$.

313 tumefaciens C58 reference genome, but is annotated by the MicroScope database as

314 AERS4k1_1608 (Vallenet et al., 2009), and in the recent re-annotation of the $A$.

315 tumefaciens C58 genome as ATU_RS08050. We hypothesized that the protein product of ATU_RS08050 may be required for the gain of motility in our isolated suppressors of $\Delta e x o R$. As detailed below, we have now designated this gene as the mirA. To test whether mirA is necessary for the non-motile phenotype of $\Delta e x o R$ mutants, we constructed an in-frame deletion mutant in both the wild type and $\Delta e x o R$ backgrounds.

320 We observed wild-type motility in both the $\triangle$ mirA and $\triangle$ exoR $\Delta$ mirA strains (Fig. 3B), 321 suggesting that this gene is necessary for motility inhibition by the ExoR-ChvG-Chvl 322 system. Given our prior observations that the ExoR-ChvG-Chvl pathway inhibits expression of class II flagellar genes (Heckel et al., 2014), that are activated by Rem

324 (Rotter et al., 2006), we tentatively designated the gene as mirA, for motility inhibitor of 325 Rem. To test whether translation of mirA is required for the non-motile phenotyoe of 
$\triangle$ exoR, we replaced the start codon ATG with a nonfunctional ATC; this mutation suppressed the $\triangle$ exoR motility defect (Fig. S10C). Additionally, introduction of the amber stop codon TAG into the mirA reading frame (following glutamic acid 20, outside of the overlap with the initially annotated Atu1638 coding sequence) also suppressed the $\triangle$ exoR motility defect (Fig. S12B), indicating that translation of the mirA gene product is specifically required for motility inhibition in $\triangle e x o R$ mutants.

\section{Expression of mirA is sufficient to inhibit motility and is under ExoR-ChvG-Chvl} control

Multiple deep sequencing studies have identified a small RNA adjacent to, and overlapping with the mirA gene $(1,625,555-1,625,785$ bp on the C58 circular chromosome); these sRNAs detected by separate studies are RNA353 (140 bp, from position1,625,422-1,625,562), an unnamed sRNA of 214 bp (from position 1,625,4251,625,639, and C1_1625426F (469 bp, from position 1,625,426-1,625,895), the last of which fully overlaps the mirA gene (Dequivre et al., 2015, Lee et al., 2013, Wilms et al., 2012). In the study by Lee, et al., C1_1625426F was predicted to encode a small open reading frame, which, in this study, we are naming mirA. To test whether expression of mirA is sufficient to achieve motility inhibition, we ectopically expressed a plasmid-borne copy of the mirA coding sequence from the constitutive coliphage T5 promoter $P_{\mathrm{N} 25}$ (Wang et al., 2000). We found that ectopic expression of the mirA coding sequence strongly inhibited motility to an equal extent as observed for the $\Delta$ exoR mutation (Fig. $3 \mathrm{~B}$ ), indicating that mirA is sufficient for motility inhibition. Plasmid-borne expression of mirA from its native promoter (including the $R N A 353$ sequence) $\left(\mathrm{p} P_{\text {mirA }:: \text { mirA }}\right.$ ) also showed a similar strong motility inhibition in a wild-type background (Fig. S13),. 
Expression from this plasmid also complemented the $\triangle e x o R \Delta m i r A$ strain, bringing

motility back to the level of the $\Delta$ exoR strain.

To determine whether the expression mirA is dependent on the ExoR-ChvG-Chvl pathway, we fused the promoter and start codon of mirA to lac $Z$ and performed a $\beta$ galactosidase assay at $\mathrm{pH} 7$ and 5.5 . We found that activity of $P_{\text {mirA-lacZ }}$ was elevated in the $\triangle e x O R$ mutant background and to a lesser extent in cells grown in $\mathrm{pH} 5.5$ medium relative to $\mathrm{pH} 7$ medium (Fig. $2 \mathrm{C}$ ). Although we did not observe a change in mirA expression in our previous transcriptomic analysis of $\Delta$ exoR compared with wild type

357 (Heckel et al., 2014), this is unsurprising as mirA was not previously annotated as a gene and was not included in the gene array that was used in that study. Expression of mirA was not affected by a frameshift mutation within the native mirA gene (Fig. S10D), indicating that mirA is not autoregulatory. To test for regulation of MirA protein levels, we replaced the native copy of mirA with a C-terminal, in-frame $\mathrm{FLAG}_{3}$ tag fusion. We

362 found that levels of MirA-FLAG 3 were elevated in $\triangle$ exoR (Fig. 2D), further evidence that MirA is regulated by the ExoR-ChvG-Chvl system.

\section{MirA regulates motility gene expression}

hypothesized that cells expressing mirA would be decreased for class II motility gene motility gene expression and to uncover genes regulated by MirA, we performed RNA- 
371 same plasmid with mirA expressed from the $P_{\text {mirA }}$ native promoter. A total of 131 genes

372 were differentially expressed ( $\log _{2}$ fold change $<-0.50$ or $>0.50$ and $p<0.05$ )(Table S1,

373 Fig. 4). The majority of these genes had decreased expression in cells ectopically

374 expressing mirA (112 genes), and we observed elevated expression of a much smaller

375 subset of the genes (19 genes). In strains harboring the mirA plasmid, expression of

376 genes of the flagellar gene clusters and chemotactic genes were strongly down-

377 regulated (Table S1; Fig. 4, blue circles). Since $\Delta$ exoR mutants are non-motile but

378 retain wild-type expression of flagellar gene activators visNR and rem (Fig. S3) (Heckel

379 et al., 2014), we hypothesized that strains with elevated mirA expression would not lead

380 to changes in expression of these genes. Indeed, we observed that expression of vis $N$,

381 visR, and rem were not significantly altered by expression of mirA (Table S1).

382 Comparison of the transcriptomes from $\Delta$ exoR vs wild type with the derivative with

383 ectopic mirA expression reveals that most genes in the MirA regulon are a subset of

384 genes regulated by ExoR and VisR, with 96 genes differentially regulated when

385 comparing $\triangle e x o R$ and/or $\triangle$ exoR to wild type ( $\log _{2}$ fold change $<-0.50$ or $>0.50$ and

p<0.05)(Table S2)(Heckel et al., 2014, Xu et al., 2013). A gene cluster that was uniquely

- Atu1194 (ATU_RS05840- ATU_RS05900). However, expression of these genes was

extremely low in cells harboring both pmirA and the empty vector, so the importance

390 and relevance of this cluster is unknown. Expression of the T6SS genes icmF and impl

391 (ATU_RS020285 and ATU_RS020300, respectively) was slightly elevated in the strain

392 harboring the mirA plasmid, and expression of these genes is also elevated in the

$393 \Delta$ exoR mutant (Heckel et al., 2014). As we have demonstrated that His6-Chvl ${ }^{\mathrm{D} 52 \mathrm{E}}$ binds 
394 at the promoter adjacent to this locus $\left(P_{h c p}\right)($ Fig. $2 \mathrm{~B})$, it is surprising that MirA may also

395

396

397

398

399

400

401

402

403

404

405

406

407

408

409

410

411

412

413

414

415

416 regulate expression of these genes. Mutation of mirA does not affect chvl expression (Fig. S10B), so the increase in T6SS gene expression is unlikely to be due to elevated chvl expression upon mirA induction. Similarly, the increase in expression of ATU_RS20300 cannot simply be explained as a decrease in Rem-dependent gene activation because expression of ATU_RS20300 is decreased in a $\Delta v i s R$ mutant, where rem is not expressed (Heckel et al., 2014).

\section{MirA interacts with Rem in vitro}

We hypothesized that MirA dampens motility gene expression through sequestration of the motility activator protein Rem. To test whether MirA interacts directly with Rem, we performed a pull-down assay with purified untagged Rem and MirA fused to a C-terminal hexahistidinyl tag (MirA-His6). We used cobalt resin, which interacts strongly with hexahistidinyl tags (Lichty et al., 2005), for a pull down assay. Rem did not bind the cobalt resin alone, but co-eluted with MirA-His 6 following coincubation of the proteins (Fig. 5A). We also performed a farwestern experiment to separately test MirA-Rem interaction. Dilutions of purified Rem were separated by SDSPAGE and transferred to a nitrocellulose membranes, and following blocking, these were incubated with blocking agent either with or without purified MirA-His6. Bound MirA-His 6 was detected with anti-His 6 monoclonal antibodies. The strength of anti-His 6 chemiluminescent signal correlated with the concentration of Rem protein and depended on the presence of MirA-His6 (Fig. 5B). 


\section{Bacterial two-hybrid analysis of MirA-Rem interactions}

We used the Bacterial Adenylate Cyclase Two-Hybrid (BACTH) assay to further assess interaction between Rem and MirA fusion proteins expressed in E. coli. This assay detects protein-protein interactions that bring the T25 and T18 fragments of

421 adenylate cyclase into close enough proximity to become catalytically active to synthesize cyclic AMP (cAMP) synthesis (Battesti \& Bouveret, 2012). cAMP synthesis activates expression of lacZ which leads to blue colonies in the presence of X-gal. Co-

424 expression of the MirA-T25 and Rem-T18 fusions resulted in blue colonies, further 425 supporting a direct interaction between MirA and Rem (Fig. 5C). A similar result was

426 obtained for the reverse combination Rem-T25 and MirA-T18, while negative controls

427 harboring a sole MirA- or Rem- adenylate cyclase fragment yielded negative results, 428 indicated by white colonies. Strains expressing MirA-T25 and MirA-T18 or Rem-T25 and 429 Rem T18 also resulted in blue colonies, suggesting self-interactions of MirA and Rem, 430 in addition to their formation of a MirA-Rem heterocomplex. As with many two-

431 component-type response regulator transcription factors (Gao et al., 2007), the Rem protein contains a receiver domain and DNA-binding domain. To assess which domain of Rem interacts with MirA, we tested the receiver and DNA-binding domains

434 individually for interaction with MirA via BACTH. We observed a strong interaction of 435 MirA with the DNA-binding domain of Rem, and little to no interaction of MirA and the 436 receiver domain (Fig. 5C). 


\section{MirA inhibits Rem-DNA binding}

Given our BACTH data supporting a model where MirA interacts with the Rem DNA-binding domain, we hypothesized that this interaction might impair Rem DNAbinding activity. To test this model, we co-incubated purified Rem and MirA-His 6 protein prior to incubation with a target promoter of Rem, $P_{\text {motA, }}$ and performed electrophoretic mobility shift assays (Fig. 6A). Co-incubation with increasing concentrations of MirA-His6 led to disruption of Rem-DNA binding, even at molar ratios of the two proteins at or below one-to-one. To test whether MirA-His6 could disrupt pre-formed complexes between Rem and $P_{\text {motA }}$ promoter DNA, we incubated Rem with the promoter DNA to allow complexes to form, and then added MirA-His 6 at varying concentrations. As the concentration of MirA-His 6 increased, we observed an increase in free DNA relative to the Rem-DNA complex (Fig. 6B). To test whether DNA-binding inhibition by MirA was specific to Rem, we co-incubated MirA-His6 with His6-Chvl ${ }^{\mathrm{D} 52 \mathrm{E}}$ and assessed DNA binding by Chvl (Fig. S14). Even at concentrations of MirA-His 6 that inhibited Rem-DNA binding activity, His6-Chvl ${ }^{\mathrm{D} 52 \mathrm{E}}$ was still able to bind the $P_{h c p}$ promoter DNA, suggesting that MirA inhibition of DNA binding is specific to Rem and not an inherent property of MirA.

\section{Conservation of MirA across the Rhizobiaceae: A. tumefaciens MirA inhibits} motility in S. meliloti.

The ExoR-ExoS-Chvl and the VisNR-Rem pathways have been well studied in S. meliloti (Chen et al., 2009, Rotter et al., 2006, Sourjik \& Schmitt, 1996, Wells et al., 2007). We asked whether MirA functions similarly in S. meliloti. The A. tumefaciens 
463 mirA (mirAAt) MirA was expressed in S. meliloti and the S. meliloti mirA (SM_17530,

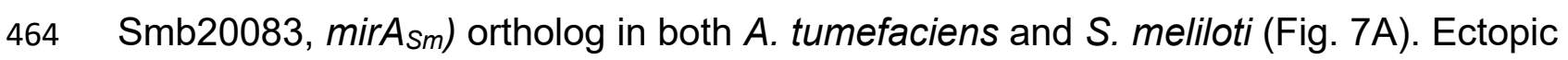

465 expression of mirA $_{A t}$ inhibited motility in both species, but mir $A_{S m}$ did not strongly alter

466 motility in either species. We hypothesized that the strain of S. meliloti tested may have

467 acquired mutations in mirA that decrease the protein's interactions with both Rem ${ }_{S m}$

468 (SM_RS03450, Smc03046) and Rem $A$. To test whether MirAsm interacts with Rem, we

469 performed BACTH assays (Fig. 7B). The BACTH results suggested MirAsm interacts

470 with itself as well as MirA $A_{A t}$. Weak interactions were observed between MirAsm and

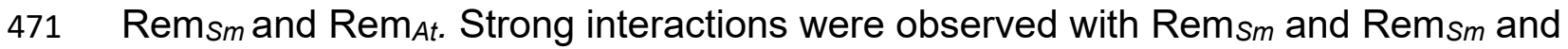

$472 \operatorname{Rem}_{A t}$, as well as MirA $A t$ with Rem ${ }_{S m}$. We hypothesized that the Rem proteins may have

473 a stronger sequence and structural conservation between $A$. tumefaciens and S. meliloti

474 than the MirA homologs. Sequence alignments of homologs from different Rhizobiales

475 species revealed that indeed, Rem proteins are highly similar across the Rhizobiales

476 order (Figure S1). Significantly greater sequence divergence is observed when

477 comparing MirA proteins from different species (indicated by asterisks)(Fig. S15). Since

478 MirA from $A$. tumefaciens binds MirA $_{A t}$ and MirA ${ }_{S m}$ and inhibits motility in both species,

479 we hypothesized that the Rem proteins are similar enough that they would be able to

480 cross-complement the rem mutants of each species. Indeed, introduction of remsm into

481 the $A$. tumefaciens $\Delta$ rem and rem $_{A t}$ into S. meliloti $\Delta$ rem complemented the motility

482 defect of the rem deletion for both species, indicating that the rem genes can cross-

483 complement (Fig. S16). 


\section{Discussion}

In this study, we investigated the mechanism by which the ExoR-ChvG-Chvl pathway inhibits flagellar motility in A. tumefaciens. We identified a new regulator of flagellar gene expression in A. tumefaciens, a previously unannotated gene that encodes a 76-aa protein we have designated MirA. We find that MirA strongly inhibits motility gene expression (Fig. 4, Table S1), but that it does not affect transcription of the motility master regulators vis $N$, visR, and rem. Instead, MirA forms a complex with the DNA-binding domain of the response regulator Rem, and this interaction inhibits the association of Rem with DNA (Fig. 5-6). Transcriptome data indicate that elevated mirA levels decrease transcription of the motility genes which are activated by Rem. We

496 believe the different methods employed in our previous studies sufficiently explain the few MirA-regulated genes that were not identified as part of the VisR regulon (and

498 therefore also the Rem regulon) by microarray (Heckel et al., 2014) (Table S2). Our data suggest that the primary function of MirA is to inhibit expression of motility genes in through activation of mirA expression (Fig. 8).

A new control mechanism for motility control in A. tumefaciens. Bacteria tightly regulate flagellar motility gene expression, only expressing genes for the components

505 required as the flagellar nanomachine is being assembled. Flagellar gene expression is sRNAs (Osterman et al., 2015). A well-characterized inhibitory control mechanism is 
activity of motility-specific sigma factors required for transcription of late stage promoters including those that control expression of flagellin genes (Calvo \& Kearns, 2015, Hughes et al., 1993). FlgM is secreted through the fully assembled type III secretion system flagellar nanomachine, releasing the cytoplasmic sigma factor to direct late-stage flagellar gene expression, thereby coupling biogenesis of the flagellar basal body to production of the flagellar filament. Although no specific sigma factor has been identified in the agrobacteria or rhizobia, the promoter elements for the class II flagellum biosynthetic genes suggest such an alternate sigma factor exists (Rotter et al., 2006). Initially, we hypothesized that MirA might function as an anti-sigma factor, but our results clearly show that MirA blocks flagellar assembly prior to class II gene expression through interaction with the Rem transcription factor.

We propose to add MirA to a small but growing list of post-transcriptional negative regulators of flagellar gene master regulators. In Salmonella enterica, YdiV is a regulatory protein that physically interacts with the FlhD flagellar activator protein, and can effectively remove the $\mathrm{FlhD}_{4} \mathrm{C}_{2}$ motility master regulatory complex from its target DNA in a similar fashion to how MirA can interfere with Rem and its ability to activate target promoters (Wada et al., 2011, Takaya et al., 2012). In contrast to MirA, YdiV is a much larger protein (237 aa) with a separate EAL domain found in phosphodiesterases that typically degrade c-di-GMP, although YdiV does not bind or turnover this second messenger (Simm et al., 2009). Interestingly, despite its larger size, YdiV acts to target the $\mathrm{FlhD}_{4} \mathrm{C}_{2}$ complex for proteolysis by $\mathrm{ClpXP}$, in addition to blocking $\mathrm{FlhD}_{4} \mathrm{C}_{2}$ from binding to its target promoters. We cannot exclude the possibility that MirA functions as an adaptor for proteolysis of Rem in addition to inhibiting its DNA binding activity. 
532 Another post-transcriptional, negative regulator of the $\mathrm{FlhD}_{4} \mathrm{C}_{2}$ complex in $\mathrm{S}$. enterica,

533 FliT, interacts with FlhC, inhibiting motility by sequestering this regulatory component

534 (Imada et al., 2010, Yamamoto \& Kutsukake, 2006). Similar to MirA, FliT self-interacts

535 (Imada et al., 2010). FliT acts as a secretion chaperone through interactions with

536 filament cap protein FliD and interacts with the chaperone export protein FliJ (Evans et

537 al., 2006). We have not observed any additional roles for MirA beyond interaction with

538 the A. tumefaciens motility regulator Rem, but additional binding partners of MirA are

539 possible.

In the aquatic APB Caulobacter crescentus, biogenesis of late-stage components

541 of the polar flagellum is regulated by the MadA-FliX-FlbD system (Siwach et al., 2021).

542 FliX is a 144-aa trans-acting regulator of flagellar gene transcription, and directly

543 interacts with the FlbD motility regulator (Xu et al., 2011). FliX also associates with a

544 gating component of the flagellar export machinery, FlhA, blocking export of late-stage

545 flagellar substrates including the flagellins. The 91-aa protein MadA binds to the export

546 machinery and stimulates displacement of FliX from FlhA, releasing it to interact with

547 FlbD (Siwach et al., 2021). Interactions with FliX are required for FlbD to function, but at

548 elevated levels also inhibit its DNA binding activity (Xu et al., 2011). In contrast, our

549 results indicate that MirA is not required for Rem activity, but rather acts to strictly inhibit

550 its ability to bind DNA. Additionally, MirA blocks expression of all the motility genes

551 downstream of VisNR and Rem, rather than specifically affecting late-stage genes.

553 control, there are multiple examples of negative regulators, anti-repressors and anti-

554 activators that directly interact with DNA-binding transcription factors to modulate their 
555 activities. In A. tumefaciens, the TraR quorum sensing transcriptional regulator is

556 inhibited through direct interactions with its anti-activator TraM (Swiderska et al., 2001).

557 TraM forms higher-order complexes with TraR dimers, blocking their ability to bind DNA

558 and targeting TraR for proteolysis (Chen et al., 2007, Chen et al., 2004, Costa et al.,

559 2012). Our BACTH results provide evidence that Rem and MirA both self-multimerize,

560 but it is not yet clear whether these putative multimers dissociate during formation of the

561 MirA-Rem complex, or rather assemble into higher-order complexes such as observed

562 for TraM-TraR.

Convergence of the ExoR-ChvG-Chvl and VisNR-Rem pathways.

In our efforts to define the inhibitory mechanism of ExoR-ChvG-Chvl on motility, we gained insight into Rem and Chvl-dependent gene regulation in A. tumefaciens.

567 Rem and its orthologs in other Rhizobiales have glutamate residues at the position that typically contains a phospho-accepting aspartate residue in two-component-type response regulators. It seemed plausible that the glutamate residue was acting as a stable phoshomimic, similar to what has been observed for other two-component

571 response regulators (Klose et al., 1993). Mutation of this glutamate residue to

572 asparagine did not abrogate Rem activity, nor did mutation of another aspartate

573 proximal to this site (D45, Fig. S5). Similarly, substitution of single aspartate residues

574 proximal to the glutamate residue at the predicted phosphorylation site of Rem $S m$ does

575 not abolish Rem activity (Rotter et al., 2006). Cumulatively, these results suggest that

576 Rem activity may not be regulated by phosphorylation, that the protein may be 
50. Additionally, we found that, unlike other aspartate-less receiver domain-containing response regulators such as RitR (Glanville et al., 2018, Maule et al., 2015), Rem does not require the cysteine at amino acid 143, as replacement of the cysteine residue with serine, aspartate, or alanine did not impact Rem function (Fig. S6). Mutation of the Chvl predicted phosphorylation site, however, did alter Chvl function in vivo. Mutation of the conserved aspartate residue at this position to asparagine (D52N) resulted in a loss-of-function allele, whereas mutation to a glutamate residue (D52E) acted as a phospho-mimic allele (Fig. 1), similar to other response regulators (Klose, 1993 \#2031). Consistent with our expectations for a phospho-mimic Chvl allele, purified His6-ChvID52E binds to a target promoter $\left(P_{h c p A}\right)$ DNA in vitro (Wu et al., 2012). Unexpectedly, we found that purified wild-type His6-Chvl, which we assumed to be un-phosphorylated, was also able to bind $\mathrm{P}_{h c p}$ in our assays (Fig. 2B). DNA binding in vitro by non-phosphorylated response regulators is not unheard of for two-component systems. In S. meliloti, the chvID52E allele has been shown to elevate succinoglycan production as detected by Calcofluor white fluorescence (Chen et al., 2009), which we also observed for A. tumefaciens (Fig. S2).

The ExoR-ChvG-Chvl system is a pervasive regulatory module in the Rhizobiales and has been proposed to regulate the free-living to host invasion switch (Heavner et al., 2015). MirA may represent a fourth component of this module, facilitating the motility regulation during this transition. Our results indicate that mirA expression is elevated in mutants expressing the chvID52E phosphomimic or in the $\Delta e x o R$ mutant. Previous analysis of the $\Delta$ exoR transcriptome did not identify mirA as an ExoR-regulated gene (Heckel et al., 2014), but mirA was not yet annotated in the $A$. 
601 tumefaciens C58 reference genome at that time. Expression of mirA is elevated in

602

603

604

605

606

607

608

609

610

611

612

613

614

615

616

617

618

619

620

621

622

623

acidic conditions. Although the ExoR-ChvG-Chvl pathway is recognized to enable

response to acidic $\mathrm{pH}$ in $A$. tumefaciens and its relatives, there is growing evidence that this pathway may provide a more general stress response, and thus MirA-dependent control of motility may be relevant under a wider set of conditions than strictly acidic $\mathrm{pH}$.

Multiple lines of evidence we present here indicate that in A. tumefaciens Chvl does not regulate vis $N$, visR, or rem gene expression, nor does it bind directly to Rem nor the promoters of motility genes (Fig. 1B, Fig. S3, Fig. 2D, Fig. S8). Rather, Chvl inhibits motility through activating expression of mirA. In S. meliloti, Chvl has been reported to directly regulate rem expression, in contrast to our findings in $A$. tumefaciens (Heckel et al., 2014, Wells et al., 2007, Ratib et al., 2018). Ectopic expression of the S. meliloti mirA (SM_RS17530, Smb20083, MirAsm) homolog did not inhibit motility in either S. meliloti or A. tumefaciens (Fig. 7A), whereas ectopic expression of mirA At inhibited motility in both bacterial taxa. The inability of MirAsm to inhibit motility may be reflective of its high sequence divergence relative to other mirA homologs (Fig. S15) and is consistent with its weak interaction with Rem from either S. meliloti or $A$. tumefaciens (Fig. 7C). It is interesting that control of motility by ExoR-ChvG-Chvl has been conserved between A. tumefaciens and S. meliloti, although the endogenous regulatory mechanism employed seems to have diverged. We observed self-interaction

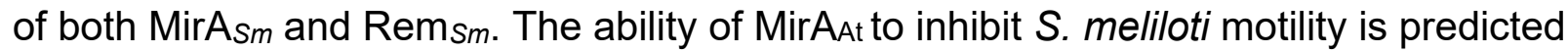
based on the high level of sequence conservation between the RemAt and Remsm proteins (Fig. 7b, Fig. S1). There are putative, better conserved MirA homologs in multiple other Rhizobiales species which also encode the Rem transcription factor (Fig. 
624 S1), suggesting that similar mechanisms of MirA-directed motility control exist in these systems.

626

627 Experimental Procedures

\section{Bacterial strains, plasmids, and oligonucleotides}

629 Bacterial strains and plasmids are listed in Table S3, and oligonucleotides are listed in

630 Table S4. E. coli strains were grown in LB at $37^{\circ} \mathrm{C}$ and $A$. tumefaciens strains were 631 grown in LB and AT minimal medium with $15 \mathrm{mM}\left(\mathrm{NH}_{4}\right)_{2} \mathrm{SO}_{4}$, buffered to $\mathrm{pH} 7$ with

$632 \mathrm{KH}_{2} \mathrm{PO}_{4}$ to a final concentration of $79 \mathrm{mM}$ with $0.5 \%$ glucose as the carbon source 633 (ATGN). Acidic medium was prepared with a final concentration of $20 \mathrm{mM}$ MES (2- $(N-$ 634 morpholino)ethanesulfonic acid) hydrate buffered to $\mathrm{pH} 5.5$ in place of $\mathrm{KH}_{2} \mathrm{PO}_{4}$ buffer. 635 S. meliloti strains were grown in YTC media (0.5\% tryptone, $0.3 \%$ yeast extract, .08\%

$\left.636 \mathrm{CaCl}_{2} \cdot 2 \mathrm{H}_{2} \mathrm{O} \mathrm{pH} 7.0\right)$. Growth temperatures were $28-30{ }^{\circ} \mathrm{C}$ for $\mathrm{S}$. meliloti and $A$.

637 tumefaciens and $37^{\circ} \mathrm{C}$ for E. coli. Antibiotics were used at the following concentrations 638 for liquid media: Kanamycin (Km) $150 \mu \mathrm{g} / \mathrm{mL}$, Gentamycin (Gm) $100 \mu \mathrm{g} / \mathrm{mL}$,

639 Carbenicillim (Cb) $25 \mu \mathrm{g} / \mathrm{mL}$, Spectinomycin (Sp) $150 \mu \mathrm{g} / \mathrm{mL}$ for A. tumefaciens; Km 25 $640 \mu \mathrm{g} / \mathrm{mL}$, Gm $25 \mu \mathrm{g} / \mathrm{mL}$, Ampicillin (Ap) $100 \mu \mathrm{g} / \mathrm{mL}$, Sp $25 \mu \mathrm{g} / \mathrm{mL}$ for E. coli; Gm $40 \mu \mathrm{g} / \mathrm{mL}$ 641 for S. meliloti.

\section{Allelic replacement and marker-less deletion construction}

644 Marker-less deletion strains was performed as previously described (Morton \& Fuqua, 645 2012). Fragments upstream and downstream of the genes of interest (0.5-1 kb in size) 646 were amplified using primers P1 and P2 (upstream) and P3 and P4 (downstream) 
647 (Table 2). These fragments were stitched together via 18-20 base-pair complementary sequences by a SOEing reaction. The deletion or allelic replacement construct was first ligated into the pGEM T-Easy vector for amplification in E. coli strain Top $10 \mathrm{~F}$.' Miniprepped pGEM plasmids were digested with the appropriate enzymes and the insert

651 ligated into the suicide vector pNPTS138 (Hibbing \& Fuqua, 2011). This vector was

652 transformed into the $E$. coli conjugal donor strain $\mathrm{S} 17-1 / \lambda$ pir to transfer the deletion 653 plasmid into the appropriate $A$. tumefaciens strain. Mixtures of $A$. tumefaciens recipient 654 and E. coli donor strains were spotted onto cellulose acetate filter disks as described 655 previously (Morton \& Fuqua, 2012). Primary integrants were selected by plating cells collected from filter disks onto ATGN-Km plates. Cells that subsequently lost the

657 integrated plasmid but retained the introduced deletion were isolated through passage 658 in the absence of antibiotic by plating onto AT medium with $5 \%$ sucrose instead of 659 glucose (ATSN) and confirmed by PCR across the deletion junction.

\section{Construction of expression plasmids.}

662 Controlled expression plasmids were generated by introducing $A$. tumefaciens coding sequences amplified from genomic DNA by PCR into the Lacl ${ }^{\mathrm{Q}}$-encoding, IPTG

664 (isopropyl- $\beta$-D-thiogalactopyranoside)-inducible expression vector with a gentamicin 665 resistance $\left(\mathrm{Gm}^{\mathrm{R}}\right)$ cassette, pSRKGm (Khan et al., 2008). For expression mirA from its

666 native promoter and $P_{l a c}$, the mirA coding sequence and upstream region were cloned 667 into pSRKGm at the Ndel site, with a stop codon to prevent translation across the Ndel 668 site from the upstream lacZ $\alpha$. To construct $P_{\text {mirA }}:$ mirA $A_{s m}$, the mirA gene from $A$. 
670 expression from the phage promoter $P_{\mathrm{N} 25}$, mirA was cloned into pYW15c (Wang et al.,

671 2000). Coding sequences were amplified from $A$. tumefaciens C58 genomic DNA or

672 plasmids carrying engineered alleles using the 5' and 3' primers corresponding to each

673 gene, specified in Table S4. Amplified fragments were ligated into the cloning vector

674 pGEM-T Easy (Promega), or built directly into the plasmid of interest using the

675 NEBuilder HiFi Assembly kit (New England Biolabs), transformed into E. coli Top10 F'

676 or $\mathrm{DH} 5 \alpha / \lambda$ pir for amplification and collection, and the inserts were confirmed by

677 sequencing. Coding sequences were excised from the pGEM-T Easy cloning vectors by

678

restriction enzyme cleavage and ligated into the appropriately cleaved pSRKGm vector

679

using T4 DNA ligase (New England Biolabs). Derived plasmids were verified by PCR

680

amplification across the multiple cloning site and insert prior to transformation into $A$.

681

tumefaciens cells via electroporation (Mersereau et al., 1990).

682

\section{Site-directed mutagenesis of Chvl and Rem}

Site-directed mutagenesis was performed to create the alleles chvID52E, chvID52N,

remD45N, remE50N, remC143S, remC143D, and remC143A. Mutagenesis was

performed as previously described (Hibbing \& Fuqua, 2011, Mohari et al., 2018) using

687 the protocol described in the QuickChange Site-Directed Mutagenesis Kit (Stratagene

688 Corp) or the Q5 Site-Directed Mutagenesis Kit (New England Biolabs). The desired

689 nucleotide changes were designed into the complementary mutagenesis primers (Table

690 S4). Mutant alleles were amplified from cloning constructs carrying the wild-type alleles.

691 After amplification of the entire plasmid, template and hemi-methylated plasmid DNA 
693 transformed into E. coli Top10 F' or DH5a/Apir, collected, sequence verified, and the 694 alleles were fused with controlled expression plasmids or allelic replacement plasmids.

\section{Construction of lacZ fusions and $\beta$-galactosidase assays}

697 Promoter fusions to lacZ were constructed by amplification of promoter regions from genomic DNA with the specified primers (Table S4) and ligated into pGEM-T Easy cloning constructs and ligated into reporter plasmid pRA301 (Akakura \& Winans, 2002) to generate reporter constructs with the promoter region, ribosome binding site, and start codon of the gene of interest fused in-frame with the second codon of the lacZ gene to generate translational fusions. glycerol at OD600 12. Thawed samples were inoculated at OD600 0.05 in $2 \mathrm{~mL}$ cultures in triplicate, grown to exponential phase at $30^{\circ} \mathrm{C}$, measured for $\mathrm{OD}_{600}$ and frozen. Thawed samples were incubated in Z buffer (Miller, 1972) with 2 drops of 0.02\% SDS, 4 drops of chloroform, vortexed for 10 seconds, and incubated with $100 \mu \mathrm{L} 4 \mathrm{mg} / \mathrm{mL}$ ortho-

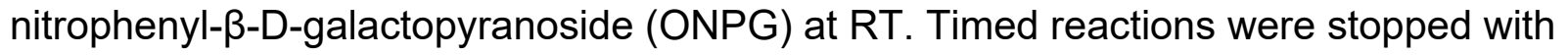
addition of $600 \mu \mathrm{L} \mathrm{NaCO}$ and clarified through centrifugation. $\beta$-galactosidase cleavage

711 of ONPG was measured through $\mathrm{A}_{420}$ measurement in a BioTek plate reader and 712 specific activity was measured in Miller Units (Miller, 1972). 


\section{5'RACE mapping transcriptional start sites}

717 Total RNA was extracted from either wild-type A. tumefaciens (for

mapping the TSS of $\operatorname{mot} A$ and $f(g B$ ) or $\triangle e x o A \triangle e x o R$ (for mapping the TSS of

hcp, chvl, and aopB). RNA was extracted using the RNeasy Midi Kit from

TURBODNA-freeтм Kit from Thermo Fisher Scientific (formerly Life Technologies).

722 Enzymes provided in the TAKARA 5'-Full RACE Core Set were used for 1st

723 strand cDNA synthesis, degradation of hybrid DNA-RNA, and circularization of instructions. Amplified promoter fragments were ligated into the pGEM-T Easy

\section{RNA sequencing} was purified with the Qiagen RNEasy kit, and DNA was digested with the Turbo RNAsefree DNAse kit for $1 \mathrm{hr}$ at $37^{\circ} \mathrm{C}$. The integrity of the RNA was checked with Agilent

735 Tapestation. From each sample, 1 ug of total RNA was used for ribosomal RNA 736 depletion with the Ribo-Zero ${ }^{\mathrm{TM}}$ Magnetic Kit (Bacteria)(Epicenter). The libraries were

737 then prepared with the TruSeq Stranded mRNA library preparation kit (Illumina). The 
739 high cycle sequencing module to generate paired-end reads. Mutations were identified

740

741

742

743

744

745

746

747

748

749

750

751

752

753

754

755

756

757

758

759

760

761

by mapping reads against the $A$. tumefaciens C58 reference genomes (Genbank accession numbers AE008687, AE008688, AE008689, and AE008690 for the circular, linear, At, and Ti plasmids, respectively) using the breseq computational pipeline (Deatherage \& Barrick, 2014). Transcriptome data is summarized in Table S1 and is available at GEO-NCBI (GSE174467).

\section{Motility assays}

To quantify the efficiency of flagellar locomotion, strains were inoculated into the center of motility medium plates with $0.3 \%$ Bacto Agar suspended in media (ATGN for $A$. tumefaciens, Bromfield agar (Sourjik, 1996 \#1979) for S. meliloti) and the diameter of each swim ring was measured over time. Strains were inoculated from single colonies from $1.5 \%$ agar (ATGN or $2 x Y T$ ) plates with a toothpick or wire. Swim plates were incubated at RT in a sealed container with an open vial of a saturated $\mathrm{K}_{2} \mathrm{SO}_{4}$ solution to maintain a relative humidity of $\sim 97 \%$ in the chamber. Swim ring diameters were measured with a standard ruler.

\section{Biofilm assays}

Biofilm assays were performed as previously described (Tomlinson et al., 2010).

Cultures of $A$. tumefaciens were inoculated at an initial OD600 0.05 in ATGN

supplemented with $22 \mu \mathrm{M} \mathrm{FeSO} 4 \cdot 7 \mathrm{H}_{2} \mathrm{O}$ into 12-well plates with upright polyvinyl chloride (PVC) coverslips or into 96-well PVC plates for large-scale assays. Plates were incubated at RT 24-48 h in a sealed container with an open vial of a saturated solution of $\mathrm{K}_{2} \mathrm{SO}_{4}$ to maintain a relative humidity of $\sim 97 \%$ in the chamber. The coverslips or wells 
762 were stained with $0.1 \%$ crystal violet dye and the dye was solubilized in $33 \%$ acetic acid. Biofilm biomass was determined by measuring the A600 of solubilized crystal violet and normalizing by the OD600 of the cultures at the time of harvesting. The cell density and absorbance readings were measured with a Bio-TEK Synergy HT plate reader using Bio-TEK Gen5 (version 1.07) software.

\section{Calcofluor white staining}

ATGN plates were supplemented with $200 \mu \mathrm{g} / \mathrm{mL}$ of Calcofluor White (fluorescent white) were normalized to an OD 6000.2 , and $5 \mu \mathrm{L}$ of each culture was spotted onto the plate. Plates were incubated for 2 days at $28^{\circ} \mathrm{C}$ and then imaged with UV light excitation using

774 a Biorad ChemiDoc system with Image Lab software. All strains were grown and imaged on the same plate.

\section{Isolation and characterization of motile suppressors of $\Delta$ exoR}

779 (Heckel et al., 2014). Briefly, the nonmotile $\triangle e x o R \Delta e x o A$ mutant strain was dissolved in ATGN media. Motile suppressor mutants were harvested from the edge of

782 the swim ring. To identify suppressors with mutations beyond the chvG-chvl locus, 
sequencing to identify the site of transposon insertion for these mutants as well as whole genome sequencing to identify point mutations in these genomes.

\section{Whole genome sequencing}

Genomic DNA was prepared from $1 \mathrm{~mL}$ of cultures grown in ATGN medium to mid-log phase. Genomic DNA of the $\Delta e x o A \Delta e x o R$ parent and four suppressor mutants was prepared and used to create libraries for sequencing. Libraries were prepared using a Bio Scientific NEXTflex ${ }^{\text {TM }}$ Rapid DNA sequencing kit according to manufacturer instructions. Sequencing was performed with an Illumina Nextseq instrument with subsequent analysis from the IU CGB. Mutations were identified by mapping reads against the $A$. tumefaciens C58 reference genomes (Genbank accession numbers AE008687, AE008688, AE008689, and AE008690 for the circular, linear, At, and Ti plasmids, respectively) using the breseq computational pipeline (Deatherage \& Barrick, 2014).

\section{Expression and purification of proteins}

The rem allele was amplified from a cloning construct (primer sequences in Table S4) and ligated into pTYB12 (NEB IMPACT system of protein purification), resulting in an Nterminal intein fusion to Rem. The expression plasmid was transformed into $E$. coli Top10 F' for sequence verification and collection and then transformed into $E$. coli BL21/ADE3 for expression and purification. Expression and purification of the inteintagged Rem protein was performed according to the protocol described in the NEB IMPACTтм Kit. One liter of cells was grown at $37^{\circ} \mathrm{C}$ on an orbital shaker to an OD600 0.5 
808 then induced with IPTG to a final concentration of $400 \mu \mathrm{M}$. Cells were induced overnight on an orbital shaker at $16^{\circ} \mathrm{C}$. Cells were collected by centrifugation at $5,000 \times \mathrm{g}$ for 15 min at $4^{\circ} \mathrm{C}$, and the pellet was re-suspended in column buffer $(20 \mathrm{mM}$ Tris- $\mathrm{HCl} \mathrm{pH} 8.5$,

$811500 \mathrm{mM} \mathrm{NaCl}, 1 \mathrm{mM}$ EDTA). Cells were lysed by passage through an M-110L

812 Microfluidizer Processor (Microfluidics, Westwood,MA). Cell debris was removed by

813 centrifugation at $15,000 \times \mathrm{g}$ for $30 \mathrm{~min}$ at $4^{\circ} \mathrm{C}$ and the clarified extract was loaded onto a 814 chitin resin (NEB) column pre-equilibrated with column buffer for purification at RT. After

815 washing with 20 bed volumes of column buffer, auto-cleavage of the intein tag was

816 induced by adding 3 bed volumes of cleavage buffer (column buffer with 50 mM DTT) to

817 the column and incubating at RT for $40 \mathrm{~h}$. Un-tagged Rem protein was eluted with

818 column buffer and pooled fractions were dialyzed into a storage buffer (10 mM Tris- $\mathrm{HCl}$,

$819 \mathrm{pH}$ 8.0, $250 \mathrm{mM} \mathrm{NaCl}, 1 \mathrm{mM}$ DTT, 10\%glycerol). Aliquots were flash frozen and stored 820 at $-80^{\circ} \mathrm{C}$.

822 amplified from cloning constructs (primer sequences in Table 2) and ligated into pET28a(+) (Novagen/ EMB Biosciences/ Millipore). Protein-expression plasmids were

824 transformed into E.coli Top10 F' for collection and sequence verification and then

825 transformed into E.coli BL21/ADE3 for expression and protein purification. One liter of 826 cells were grown in LB at $37^{\circ} \mathrm{C}$ on an orbital shaker to an OD600 of 0.6 , then induced 827 with $500 \mu \mathrm{M} \mathrm{IPTG}$ for 4 hours at $37^{\circ} \mathrm{C}$. Cells were collected by centrifugation at $4^{\circ} \mathrm{C}$ at $8285,000 \times \mathrm{g}$ for $15 \mathrm{~min}$. Cell pellets containing Chvl were resuspended in lysis buffer (50 $829 \mathrm{mM} \mathrm{NaH}_{2} \mathrm{PO}_{4} \mathrm{pH}$ 6.5, 300mM NaCl, $10 \mathrm{mM}$ imidazole) with $1 \mathrm{mM}$ PMSF 830 (phenylmethanesulfonyl fluoride). Cells were lysed by passage through an M-110L 
831 Microfluidizer Processor (Microfluidics). Cell debris was removed by centrifugation at

$83215,000 \times \mathrm{g}$ for $30 \mathrm{~min}$ at $4^{\circ} \mathrm{C}$ and pellets were resuspended in storage buffer (50 mM

$833 \mathrm{NaPO}_{4} \mathrm{pH} 6.5,500 \mathrm{mM} \mathrm{NaCl}$ ). Purification was performed on $1 \mathrm{ml}$ HisTrap HP (GE

834 Healthcare) column using an FPLC system (ÄKTA P-920 pump and Superdex 75

835 10/300 GL column, GE Healthcare). Protein-containing fractions were pooled for

836 desalting through a $5 \mathrm{~mL}$ HiTrap Desalting column (GE Healthcare). Desalted protein

837 was dialyzed into a storage buffer (50 mM NaH2 $\mathrm{PO}_{4} \mathrm{pH} 8.0,500 \mathrm{mM} \mathrm{NaCl}, 20 \%$

838 glycerol). Protein concentration was calculated following absorbance measurement by

839 Nanodrop with the molar extinction coefficient for each protein.

840

Cell pellets from cells expressing MirA-His6 were thawed and suspended in 20

$\mathrm{mM} \mathrm{NaH}_{2} \mathrm{PO}_{4}, 150 \mathrm{mM} \mathrm{NaCl}, \mathrm{pH} 7.4$ buffer with $1 \mathrm{mM}$ PMSF and cells were disrupted

842

by passage through an EmulsiFlex-C3 emulsifier, and clarified by centrifugation.

843 Clarified lysates were loaded onto a $1 \mathrm{~mL}$ TALON (Takara) column, washed with $20 \mathrm{mM}$

$844 \mathrm{NaH}_{2} \mathrm{PO}_{4}, 150 \mathrm{mM} \mathrm{NaCl}, \mathrm{pH} 7.4$ buffer and eluted with $20 \mathrm{mM} \mathrm{NaH}_{2} \mathrm{PO}_{4}, 150 \mathrm{mM} \mathrm{NaCl}$,

$8455 \%$ glycerol, pH 7.4 buffer with 100-600 mM imidazole step elution fractions. Eluted

846 fractions were pooled and dialyzed into $20 \mathrm{mM} \mathrm{NaH}_{2} \mathrm{PO}_{4}, 150 \mathrm{mM} \mathrm{NaCl}, 5 \%$ glycerol,

$847 \mathrm{pH} 7.4$ buffer and frozen at $-20^{\circ} \mathrm{C}$. Protein concentration was determined by Pierce

848 BCA assay as molar absorptivity measurements with MirA-His6 were clearly erroneous.

\section{Electrophoretic mobility shift assay}

851 Upstream DNA fragments suspected to have promoter elements were amplified and

852 purified with the E.Z.N.A. Cycle Pure kit (Omega Bio-Tek). 10 nM DNA fragments were

853 incubated with purified protein in binding buffer (10 mM Tris-Cl pH 7.5, $1 \mathrm{mM}$ EDTA, 0.1 
$854 \mathrm{mM}$ DTT, $5 \%$ glycerol, and $0.05 \mathrm{mg} / \mathrm{mL} \mathrm{BSA}$ ) and incubated $20 \mathrm{~min}$ at RT. Reactions

855

856

857

858

859

860

861

862

863

864

865

866

867

868

869

870

871

872

873 identical gels (12.5\% acrylamide, 37.5:1 acrylamide:bisacrylamide) and transferred to

874

875

876 were run on $6 \%$ acrylamide (80:1 acrylamide: bisacrylamide) $0.5 \%$ TBE ( $45 \mathrm{mM}$ Tris-

borate, $1 \mathrm{mM}$ EDTA pH 8.0) gels and electrophoresed for $2 \mathrm{~h}$ at $150 \mathrm{~V}$. Prior to loading, gels were pre-run with $0.5 \%$ TBE at $75 \mathrm{~V}$ for $1 \mathrm{~h}$. Gels were stained with a $10,000: 1$ dilution of SYBR Safe DNA gel stain (Invitrogen) 20 min in 0.5X TBE, rinsed with distilled water and imaged on a Bio-Rad ChemiDoc system using Image Lab software. For gel shift inhibition assays, purified MirA-His 6 protein was incubated with Rem protein for fixed incubation times at RT prior to the addition of DNA, followed by $15 \mathrm{~min}$ incubation at RT. Reactions were run on an $8 \%$ polyacrylamide gel prior to staining for 15 min with $1 \mu \mathrm{g} / \mathrm{mL}$ ethidium bromide and imaging on a Bio-Rad ChemiDoc.

\section{Pull-down assay}

Purified proteins were diluted to the appropriate concentration in $50 \mathrm{mM}$ Tris- $\mathrm{HCl}, 100$ $\mathrm{mM} \mathrm{NaCl}, 0.1 \%$ Tween $\mathrm{pH} 7.5$ buffer. Samples were incubated $30 \mathrm{~min}$ at RT and loaded onto a $10 \mu \mathrm{L}$ aliquot of Talon (Takara) resin. Following a 30 min incubation, the resin was washed 3 times with $500 \mu \mathrm{L}$ buffer and eluted with $20 \mu \mathrm{L}$ Laemlli loading buffer.

\section{Western and farwestern blotting}

For farwestern analysis, purified protein was electrophoresed by SDS-PAGE in two nitrocellulose membranes. Membranes were blocked with $5 \%$ nonfat dry milk in TBS $0.1 \%$ Tween for 1 hour. For MirA-6His, membranes were blotted either with milk containing purified 0.4 M MirA-His6 or buffer. For ${ }_{6} H i s-C h v l^{\mathrm{D} 52 \mathrm{E}}$, the proteins on the 
877 membrane were denatured by incubation in AC buffer (100 mM NaCl, $20 \mathrm{mM}$ Tris, 0.5 mM EDTA, 10\% glycerol, $0.1 \%$ Tween-20) with $6 \mathrm{M}$ guanidine- $\mathrm{HCl}$ for 30 min at RT with mixing. Proteins were renatured by incubating at RT with shaking for $30 \mathrm{~min}$ in AC buffer with $3 \mathrm{M}$ guanidine- $\mathrm{HCl}$, then 30 min at $\mathrm{RT}$ in $\mathrm{AC}$ buffer with $1 \mathrm{M}$ guanidine- $\mathrm{HCl}$, then 30 minutes at $4^{\circ} \mathrm{C}$ in $\mathrm{AC}$ buffer with $0.1 \mathrm{M}$ guanidine- $\mathrm{HCl}$, then overnight at $4^{\circ} \mathrm{C}$ in AC buffer. The membranes were blocked in blotto ( $\% 5$ milk solution in tris-buffered saline with $1 \%$ Tween-20) at RT $1 \mathrm{~h}$ with shaking. Purified His6-ChvID ${ }^{52 E}$ protein $(5 \mu g)$ was used as prey and incubated with a membrane in protein binding buffer (AC buffer with $5 \%$ milk powder and $1 \mathrm{mM}$ DTT) overnight at $4{ }^{\circ} \mathrm{C}$ with shaking. Another membrane was processed in tandem in the same conditions with no His6-Chvl ${ }^{\mathrm{D} 52 \mathrm{E}}$. Membranes for both proteins were then incubated with a 1:2000 dilution of rabbit $\alpha$-His antibody for 1 hour at RT, then with a 1:20,000 dilution of HRP-conjugated goat anti-rabbit antibody for 1 hour at RT. For detection of MirA-FLAG 3 , strains were grown to exponential phase, concentrated to an OD600 of 10 , and boiled 5 min prior to running on a $12.5 \%$ acrylamide (37.5:1 acrylamide:bisacrylamide) SDS-PAGE gel with 2,2,2 trichloroethanol (TCE). The TCE was UV-activated prior to transfer to a nitrocellulose membrane. Membrane transfer was confirmed through UV-imaging of membrane 894 (BioRad ChemiDoc ImageLab software). The membranes were incubated with 1:5,000 monoclonal ANTI-FLAG M2 antibody produced in mouse (Sigma-Aldrich) and Goat Anti-Mouse IgG (H+L) HRP Conjugate (BioRad). Membranes were developed with signal was detected using a Biorad ChemiDoc system using Image Lab software. 


\section{Bacterial adenylate cyclase two-hybrid assays}

901 Genes of interest were PCR-amplified and inserted into Kpnl and EcoRI-digested

902 pKNT25 or pUT18 with NEB Hifi Assembly to construct in-frame C-terminal fusions

903 (Euromedex). Plasmids containing T18 and T25 fusions were co-transformed into

904 BACTH test strain BTH101. Strains were grown overnight at $30^{\circ} \mathrm{C}$ and $2 \mu \mathrm{L}$ were 905 spotted onto LB plates containing IPTG $(500 \mu \mathrm{M})$ and 5-bromo-4-chloro-3-indolyl- $\beta$-Dgalactopyranoside (X-gal, $80-160 \mu \mathrm{g} / \mathrm{mL}$ ) and incubated at $30^{\circ} \mathrm{C}$ overnight. Colonies

907 908 909

910

were imaged with a Nikon camera. All strains were grown and imaged on the same plate for each experiment.

\section{Multiple Sequence Alignment}

Amino acid sequences were retrieved from Kegg, Biocyc or JGI IMG and aligned using ClustalW with the default parameters (Gap open penalty $=10$, Gap extension penalty $=$ 0.5, BLOSUM weight matrix). Amino acid sequences of proteins can be found at NCBI reference numbers WP_006313038.1 (Rem/A. tumefaciens), CAC45250.1 (Rem/S. meliloti), SUB44985.1 (Rem/O. anthropi), WP_006698188.1 (Rem/R. pusense IRGB74), WP_065114859.1 (Rem/A. rhizogenes) WP_010970615.1 (Chvl/A. tumefaciens), WP_003531999.1 (Chvl/S. meliloti), AYM61105.1 (VirG/A. tumefaciens), NP_417864.1 (OmpR/E. coli), AKH06072.1 (PhoB/S. enterica), WP_003502959.1 (MirA/ A. tumefaciens), CAC48483.1 (MirA/ S. meliloti), WP_004442019.1 (MirA/R. pusense IRGB74), WP_065115793.1 (MirA/A. rhizogenes), and WP_040128199.1 (MirA/Ochrobactrum/Brucella anthropi). 


\section{Acknowledgements}

924 This project was supported by National Institutes of Health (NIH) grant GM120337

925 (C.F.) Illumina DNA Sequencing and bioinformatic analysis of whole genome re--

926 sequencing and RNAseq were performed by the Indiana University Center for

927 Genomics and Bioinformatics.

928

929 Author contributions: C.F. formulated the research plan; M.A.A, B.C.H., A.S., J.A.S.

930 and C.F. performed the experiments, C.F., M.A.A. and B.C.H. wrote the paper.

932 Data availability: High-throughput, Illumina-based RNASeq data has been deposited in

933 the GEO database Accession No. GSE174467 (([dataset] Alakavuklar and Fuqua, 2021)

934 (Deatherage et al, 2014)). All other data that support the findings of this study are 935 available from the corresponding author upon reasonable request. 


\section{References}

Akakura, R., and Winans, S.C. (2002) Constitutive mutations of the OccR regulatory protein affect DNA bending in response to metabolites released from plant tumors. J. Biol. Chem. 277: 5866-5874.

[dataset] Alakavuklar and Fuqua, 2021. High-throughput, Illumina-based RNASeq data has been deposited in the GEO database Accession No. GSE174467. All other data that support the findings of this study are available from the corresponding author upon reasonable request.

Battesti, A., and Bouveret, E. (2012) The bacterial two-hybrid system based on adenylate cyclase reconstitution in Escherichia coli. Methods 58: 325-334.Calvo, R.A., and Kearns, D.B. (2015) FlgM is secreted by the flagellar export apparatus in Bacillus subtilis. J Bacteriol 197: 81-91.

Chen, E.J., Fisher, R.F., Perovich, V.M., Sabio, E.A., and Long, S.R. (2009) Identification of direct transcriptional target genes of exoS/chvl two-component signaling in Sinorhizobium meliloti. J Bacteriol 191: 6833-6842.

Chen, E.J., Sabio, E.A., and Long, S.R. (2008) The periplasmic regulator ExoR inhibits ExoS/Chvl two-component signalling in Sinorhizobium meliloti. Mol Microbiol 69: 1290-1303.

Chen, G., Jeffrey, P.D., Fuqua, C., Shi, Y., and Chen, L. (2007) Structural basis for antiactivation in bacterial quorum sensing. Proc Natl Acad Sci U S A 104: 1647416479.

Chen, G., Malenkos, J., Cha, M.-R., Fuqua, C., and Chen, L. (2004) Quorum-sensing anti-activator TraM forms a dimer that dissociates to inhibit TraR. Mol. Microbiol. 52: 1641-1651.

Cheng, H.-P., and Walker, G.C. (1998) Succinoglycan production by Rhizobium meliloti is regulated through the ExoS-Chvl two-component regulatory system. $J$ Bacteriol 180: 20-26.

Chevance, F.F., and Hughes, K.T. (2008) Coordinating assembly of a bacterial macromolecular machine. Nat Rev Microbiol 6: 455-465.

Chevance, F.F., and Hughes, K.T. (2017) Coupling of Flagellar Gene Expression with Assembly in Salmonella enterica. Methods Mol Biol 1593: 47-71.

Costa, E.D., Chai, Y., and Winans, S.C. (2012) The quorum-sensing protein TraR of Agrobacterium tumefaciens is susceptible to intrinsic and TraM-mediated proteolytic instability. Mol. Microbiol. 84: 807-815.

Deatherage, D.E., and Barrick, J.E., (2014) Identification of mutations in laboratoryevolved microbes from next-generation sequencing data using breseq. In: Engineering and analyzing multicellular systems. Springer, pp. 165-188.

Dequivre, M., Diel, B., Villard, C., Sismeiro, O., Durot, M., Coppée, J.-Y., Nesme, X., Vial, L., and Hommais, F. (2015) Small RNA deep-sequencing analyses reveal a new regulator of virulence in Agrobacterium fabrum C58. Mol Plant Microbe Interac 28: 580-589.

Dressaire, C., Moreira, R.N., Barahona, S., Alves de Matos, A.P., and Arraiano, C.M. (2015) BolA is a transcriptional switch that turns off motility and turns on biofilm development. mBio 6: e02352-02314. 
Evans, L.D., Stafford, G.P., Ahmed, S., Fraser, G.M., and Hughes, C. (2006) An escort mechanism for cycling of export chaperones during flagellum assembly. Proc Natl Acad Sci, USA 103: 17474-17479.

Gao, R., Mack, T.R., and Stock, A.M. (2007) Bacterial response regulators: versatile regulatory strategies from common domains. Trends Biochem Sci 32: 225-234.

Glanville, D.G., Han, L., Maule, A.F., Woodacre, A., Thanki, D., Abdullah, I.T., Morrissey, J.A., Clarke, T.B., Yesilkaya, H., and Silvaggi, N.R. (2018) RitR is an archetype for a novel family of redox sensors in the streptococci that has evolved from two-component response regulators and is required for pneumococcal colonization. PLoS Path. 14: e1007052.

Goodner, B., Hinkle, G., Gattung, S., Miller, N., Blanchard, M., Qurollo, B., Goldman, B.S., Cao, Y., Askenazi, M., Halling, C., Mullin, L., Houmiel, K., Gordon, J., Vaudin, M., lartchouk, O., Epp, A., Liu, F., Wollam, C., Allinger, M., Doughty, D., Scott, C., Lappas, C., Markelz, B., Flanagan, C., Crowell, C., Gurson, J., Lomo, C., Sear, C., Strub, G., Cielo, C., and Slater, S. (2001) Genome sequence of the plant pathogen and biotechnology agent Agrobacterium tumefaciens C58. Science 294: 2323-2328.

Heavner, M.E., Qiu, W.-G., and Cheng, H.-P. (2015) Phylogenetic co-occurrence of ExoR, ExoS, and Chvl, components of the RSI bacterial invasion switch, suggests a key adaptive mechanism regulating the transition between free-living and host-invading phases in Rhizobiales. PloS one 10: e0135655.

Heckel, B.C., Tomlinson, A.D., Morton, E.R., Choi, J.H., and Fuqua, C. (2014) Agrobacterium tumefaciens exoR controls acid response genes and impacts exopolysaccharide synthesis, horizontal gene transfer, and virulence gene expression. J Bacteriol 196: 3221-3233.

Hibbing, M.E., and Fuqua, C. (2011) Antiparallel and interlinked control of cellular iron levels by the Irr and RirA regulators of Agrobacterium tumefaciens. J. Bacteriol. 193: 3461-3472.

Hughes, K.T., Gillen, K.L., Semon, M.J., and Karlinsey, J.E. (1993) Sensing structural intermediates in bacterial flagellar assembly by export of a negative regulator. Science 262: 1277-1280.

Imada, K., Minamino, T., Kinoshita, M., Furukawa, Y., and Namba, K. (2010) Structural insight into the regulatory mechanisms of interactions of the flagellar type III chaperone FliT with its binding partners. Proc Natl Acad Sci, USA 107: 88128817.

Kawagishi, I., Muller, V., Williams, A.W., Irikura, V.M., and Macnab, R.M. (1992) Subdivision of flagellar region III of the Escherichia coli and Salmonella typhimurium chromosomes and identification of two additional flagellar genes. $J$ Gen Microbiol 138: 1051-1065.

Khan, S.R., Gaines, J., Roop, R.M., 2nd, and Farrand, S.K. (2008) Broad-host-range expression vectors with tightly regulated promoters and their use to examine the influence of TraR and TraM expression on Ti plasmid quorum sensing. Appl Environ Microbiol 74: 5053-5062.

Klose, K.E., Weiss, D.S., and Kustu, S. (1993) Glutamate at the site of phosphorylation of nitrogen-regulatory protein NTRC mimics aspartyl-phosphate and activates the protein. J Mol Biol 232: 67-78. 
Lee, K., Huang, X., Yang, C., Lee, D., Ho, V., Nobuta, K., Fan, J.-B., and Wang, K. (2013) A genome-wide survey of highly expressed non-coding RNAs and biological validation of selected candidates in Agrobacterium tumefaciens. PLoS one 8: e70720.

Lichty, J.J., Malecki, J.L., Agnew, H.D., Michelson-Horowitz, D.J., and Tan, S. (2005) Comparison of affinity tags for protein purification. Protein Expression Purif. 41: 98-105.

Lu, H.-Y., Luo, L., Yang, M.-H., and Cheng, H.-P. (2012) Sinorhizobium meliloti ExoR Is the Target of Periplasmic Proteolysis. J Bacteriol 194: 4029-4040.

Maule, A.F., Wright, D.P., Weiner, J.J., Han, L., Peterson, F.C., Volkman, B.F., Silvaggi, N.R., and Ulijasz, A.T. (2015) The aspartate-less receiver (ALR) domains: distribution, structure and function. PLoS Pathog 11: e1004795.

Merritt, P.M., Danhorn, T., and Fuqua, C. (2007) Motility and chemotaxis in Agrobacterium tumefaciens surface attachment and biofilm formation. J. Bacteriol. 189: 8005-8014.

Mersereau, M., Pazour, G.J., and Das, A. (1990) Efficient transformation of Agrobacterium tumefaciens by electroporation. Gene 90: 149-151.

Miller, J.H., (1972) Experiments in Molecular Genetics. Cold Spring Harbor, New York.

Mohari, B., Thompson, M.A., Trinidad, J.C., Setayeshgar, S., and Fuqua, C. (2018) Multiple flagellin proteins have distinct and synergistic roles in Agrobacterium tumefaciens motility. J Bacteriol 200: e00327-00318.

Morton, E.R., and Fuqua, C. (2012) Genetic manipulation of Agrobacterium. Curr Protoc Microbiol: Unit 3D 2.

Mukherjee, S., and Kearns, D.B. (2014) The Structure and Regulation of Flagella in Bacillus subtilis. Annu Rev Genet 48: 319-340.

Okamura, H., Hanaoka, S., Nagadoi, A., Makino, K., and Nishimura, Y. (2000) Structural comparison of the PhoB and OmpR DNA-binding/transactivation domains and the arrangement of PhoB molecules on the phosphate box. $J \mathrm{Mol}$ Biol 295: 1225-1236.

Osterman, I., Dikhtyar, Y.Y., Bogdanov, A., Dontsova, O., and Sergiev, P. (2015) Regulation of flagellar gene expression in bacteria. Biochemistry (Moscow) 80: 1447-1456.

Prüß, B.M. (2017) Involvement of two-component signaling on bacterial motility and biofilm development. J Bacteriol 199: e00259-00217.

Ratib, N.R., Sabio, E.Y., Mendoza, C., Barnett, M.J., Clover, S.B., Ortega, J.A., Dela Cruz, F.M., Balderas, D., White, H., and Long, S.R. (2018) Genome-wide identification of genes directly regulated by $\mathrm{Chvl}$ and a consensus sequence for Chvl binding in Sinorhizobium meliloti. Mol Microbiol 110: 596-615.

Rotter, C., Muhlbacher, S., Salamon, D., Schmitt, R., and Scharf, B. (2006) Rem, a new transcriptional activator of motility and chemotaxis in Sinorhizobium meliloti. $J$ Bacteriol 188: 6932-6942.

Simm, R., Remminghorst, U., Ahmad, I., Zakikhany, K., and Romling, U. (2009) A role for the EAL-like protein STM1344 in regulation of CsgD expression and motility in Salmonella enterica serovar Typhimurium. J Bacteriol 191: 3928-3937.

Siwach, M., Kumar, L., Palani, S., Muraleedharan, S., Panis, G., Fumeaux, C., Mony, B.M., Sanyal, S., Viollier, P.H., and Radhakrishnan, S.K. (2021) An organelle- 
tethering mechanism couples flagellation to cell division in bacteria. Dev. Cell 56: 657-670. e654.

Sourjik, V., Muschler, P., Scharf, B., and Schmitt, R. (2000) VisN and VisR are global regulators of chemotaxis, flagellar, and motility genes in Sinorhizobium (Rhizobium) meliloti. J Bacteriol 182: 782-788.

Sourjik, V., and Schmitt, R. (1996) Different roles of CheY1 and CheY2 in the chemotaxis of Rhizobium meliloti. Mol. Microbiol. 22: 427-436.

Soutourina, O.A., and Bertin, P.N. (2003) Regulation cascade of flagellar expression in Gram-negative bacteria. FEMS Microbiol Rev 27: 505-523.

Swiderska, A., Berndtson, A.K., Cha, M.-R., Li, L., Beaudoin, G.M.J.I., Zhu, J., and Fuqua, C. (2001) Inhibition of the Agrobacterium tumefaciens TraR quorumsensing regulator: interactions with the TraM anti-activator. J. Biol. Chem. 276: 49449-49458.

Takaya, A., Erhardt, M., Karata, K., Winterberg, K., Yamamoto, T., and Hughes, K.T. (2012) YdiV: a dual function protein that targets FlhDC for ClpXP-dependent degradation by promoting release of DNA-bound FlhDC complex. Mol Microbiol 83: $1268-1284$.

Tambalo, D.D., Del Bel, K.L., Bustard, D.E., Greenwood, P.R., Steedman, A.E., and Hynes, M.F. (2010) Regulation of flagellar, motility and chemotaxis genes in Rhizobium leguminosarum by the VisN/R-Rem cascade. Microbiology 156: 16731685.

Tomlinson, A.D., Ramey-Hartung, B., Day, T.W., Merritt, P.M., and Fuqua, C. (2010) Agrobacterium tumefaciens ExoR represses succinoglycan biosynthesis and is required for biofilm formation and motility. Microbiology 156: 2670-2681.

Vallenet, D., Engelen, S., Mornico, D., Cruveiller, S., Fleury, L., Lajus, A., Rouy, Z., Roche, D., Salvignol, G., Scarpelli, C., and Médigue, C. (2009) MicroScope: a platform for microbial genome annotation and comparative genomics. Database 2009.

Wada, T., Morizane, T., Abo, T., Tominaga, A., Inoue-Tanaka, K., and Kutsukake, K. (2011) EAL domain protein YdiV acts as an anti-FlhD4C2 factor responsible for nutritional control of the flagellar regulon in Salmonella enterica serovar Typhimurium. J Bacteriol 193: 1600-1611.

Wang, Y., Mukhopadhyay, A., Howitz, V.R., Binns, A.N., and Lynn, D.G. (2000) Construction of an efficient expression system for Agrobacterium tumefaciensbased on the coliphage T5 promoter. Gene 242: 105-114.

Wells, D.H., Chen, E.J., Fisher, R.F., and Long, S.R. (2007) ExoR is genetically coupled to the ExoS-Chvl two-component system and located in the periplasm of Sinorhizobium meliloti. Mol Microbiol 64: 647-664.

Wilms, I., Overloper, A., Nowrousian, M., Sharma, C.M., and Narberhaus, F. (2012) Deep sequencing uncovers numerous small RNAs on all four replicons of the plant pathogen Agrobacterium tumefaciens. RNA Biol 9: 446-457.

Wu, C.F., Lin, J.S., Shaw, G.C., and Lai, E.M. (2012) Acid-induced type VI secretion system is regulated by ExoR-ChvG/Chvl signaling cascade in Agrobacterium tumefaciens. PLoS Pathog 8: e1002938.

Xu, J., Kim, J., Koestler, B.J., Choi, J.H., Waters, C.M., and Fuqua, C. (2013) Genetic analysis of Agrobacterium tumefaciens unipolar polysaccharide production 
reveals complex integrated control of the motile-to-sessile switch. Mol Microbiol 89: 929-948.

1123

1124

1125

Xu, Z., Dutton, R.J., and Gober, J.W. (2011) Direct interaction of FliX and FlbD is required for their regulatory activity in Caulobacter crescentus. BMC Microbiol. 11: $1-9$.

Yamamoto, S., and Kutsukake, K. (2006) FliT acts as an anti-FIhD2C2 factor in the transcriptional control of the flagellar regulon in Salmonella enterica serovar typhimurium. J Bacteriol 188: 6703-6708.

Yao, S.Y., Luo, L., Har, K.J., Becker, A., Ruberg, S., Yu, G.Q., Zhu, J.B., and Cheng, H.P. (2004) Sinorhizobium meliloti ExoR and ExoS proteins regulate both succinoglycan and flagellum production. J Bacteriol 186: 6042-6049.

Yuan, Z.C., Liu, P., Saenkham, P., Kerr, K., and Nester, E.W. (2008) Transcriptome profiling and functional analysis of Agrobacterium tumefaciens reveals a general conserved response to acidic conditions ( $\mathrm{pH}$ 5.5) and a complex acid-mediated 
1140 Figure 1. Phenotypes from chvID52E and chvID52N mutations. (A) The swimming

1141 and biofilm deficiencies of the $\triangle e x o R$ mutant are recapitulated by the ChvID52E allele.

1142 Motility was assessed as swim ring diameter in $0.3 \%$ Bacto Agar after three days of

1143 incubation in a humid chamber at room temperature (RT) and reported values are

1144 relative to wild-type C58. Biofilms were developed on PVC coverslips in ATGN media

1145 supplemented with $22 \mu \mathrm{M} \mathrm{FeSO}_{4} \cdot 7 \mathrm{H}_{2} \mathrm{O}$ for $48 \mathrm{~h}$. Biofilm biomass was determined by

1146 solubilizing $0.1 \%$ crystal violet-stained biofilm cells in $33 \%$ acetic acid and measuring

1147 the absorbance at $600 \mathrm{nM}\left(\mathrm{A}_{600}\right)$. Crystal violet absorbance was normalized per cell

1148 count by dividing by the planktonic culture OD600. The data are presented as biofilm

1149 biomass relative to wild type. Error bars represent standard deviation for at least three

1150 replicates for each strain. (B) $\beta$-galactosidase assays of $A$. tumefaciens derivatives

1151 harboring plasmid-borne lacZ fusions. Activity of the five indicated promoters was

1152 assessed by translational fusions to lacZ and measurement of $\beta$-galactosidase activity. .

1153 Cultures were inoculated from colonies and grown to exponential phase (OD600 $0.3-$

1154 0.8) for promoter activity assessment. Error bars represent standard deviation for at

1155 least three replicates.

1157 Figure 2. Electrophoretic mobility shift assay (EMSA) of DNA binding by Chvl and

1158 Rem. (A) Electrophoretic mobility shift assays were performed to evaluate binding of

$1159 \mathrm{His} 6-\mathrm{Chvl}^{\mathrm{D} 52 \mathrm{E}}$ to the intergenic region between the divergent $P_{t s s}$ and $P_{h c p}$ operons. DNA

1160 containing putative binding sites $(10 \mathrm{nM})$ was incubated with increasing concentrations 
1161 of purified His6-Chvl ${ }^{\mathrm{D} 52 \mathrm{E}}$. Binding was assessed by DNA migration of reactions run on a

$11626 \%$ polyacrylamide native gel. DNA was detected by staining with SYBR Safe dye. One

1163 discrete shift (indicated by asterisk) is observed when DNA is incubated with purified

1164 His6-Chvl ${ }^{\mathrm{D} 52 \mathrm{E}}$. The aopB promoter was used as a negative control for binding. (B) DNA

1165 binding of His6-Chvl ${ }^{\mathrm{D} 52 \mathrm{E}}$ and His-Chvl (wild-type) alleles. Upstream DNA of hcp, aopB,

1166 and chvl (10 nM) was incubated with and without $600 \mathrm{nM}$ of purified His-tagged alleles

1167 of $\mathrm{Chvl}$ and assessed for binding. (C) Binding of Rem to $10 \mathrm{nM}$ fragment with $P_{\text {motA }}$ (D)

1168 His6-Chvl ${ }^{\mathrm{D} 52 \mathrm{E}}$ does not compete for Rem- $P_{\text {motA }}$ association. His6-Chvl ${ }^{\mathrm{D} 52 \mathrm{E}}$ was incubated

1169 with Rem for 10 min prior to the addition of $10 \mathrm{nM}$ DNA to reactions and 20 min

1170 incubation prior to running samples on a $6 \%$ acrylamide gel and staining with SYBR

1171 Safe dye.

1172

1173 Figure 3. Identification of the motility inhibitor gene mirA . (A) Top arrows: gene

1174 diagram with Atu gene numbers (labeled above gene arrow) from the C58 reference

1175 genome (GenBank access. no. AE008687 for the circular chromosome) (Goodner et al.,

1176 2001). Bottom arrows: gene diagram with ATU_RS numbers (labeled above arrows)

1177 from the C58 representative genome annotation from 03-10-2020 (Genbank access. no.

1178 (NCBI-RefSeq:NC003062 for the circular chromosome). The direction of arrows

1179 indicates the direction of transcription. Stemmed circles indicate mutation sites with

1180 mutations are labeled above. The table on the right notes the genomic location

1181 (nucleotide number on C58 circular chromosome) and the resulting amino acid change

1182 for ATU_RS0850 is listed as AA change for each mutation. (B) Motility assay of mirA

1183 mutation in wild-type and $\Delta \operatorname{exO} R(\triangle e x O A)$ strains as well as wild type expressing $p P_{\mathrm{N} 25-}$ 
1184 mirA. Colonies were inoculated into the center of 0.3\% Bacto Agar and incubated for 24

$1185 \mathrm{~h}$ in a humid chamber at RT prior to swim ring measurement. Error bars represent

1186 standard deviation for at least three replicates. (C) Activity of the $P_{\text {mirA-lacZ fusion }}$

1187 following growth in neutral conditions (ATGN pH 7) or acidic medium (AT-MES pH 5.5)

1188 in wild-type and $\Delta \operatorname{exo} R(\triangle \mathrm{exo} A)$ strains. Strains were grown to exponential phase prior

1189 to freezing at $-20{ }^{\circ} \mathrm{C}$ and thawing prior to $\beta$-galactosidase assays. Error bars represent

1190 standard deviation for at least three replicates. (D) Western blot analysis of mirA-FLAG 3

1191 protein levels. Strains with the native mirA locus replaced with mirA-FLAG3 were grown

1192 to exponential phase prior to separation of whole cell lysates by SDS-PAGE $(12.5 \%$

1193 polyacrylamide gel) and transfer to a nitrocellulose membrane and probing with anti-M2

1194 antibody. Images are from a single western blot of a single gel, with identical image

1195 treatment. Bands from total protein stained with 2,2,2 trichloroethanol (TCE) are shown

1196 as a loading control.

Figure 4. Ectopic mirA expression leads to the downregulation of motility and chemotaxis genes. $\log _{2}\left(\right.$ fold change) and $-\log _{10}(p$ value) values for all $A$. tumefaciens genes from RNAseq of wild type C58 harboring an empty vector (pSRKGm) or pmirA

1201 (pMAT14, pPlac::P mirA::mirA), with three replicates per plasmid. Blue shaded dots label 1202 genes belonging to flagellar or chemotaxis gene clusters. 
1207 resin. Following a 30 min incubation and three washes to remove protein that fails to bind the resin, samples were eluted in SDS-PAGE loading buffer and aliquots were electrophoresed on a Tris-Tricine gel labeled with 2,2,2 trichloroethanol (TCE). (B)

1210 Farwestern analysis of Rem and MirA-His6. Purified Rem protein was separated by

1211 SDS-PAGE (12.5\% acrylamide) and immobilized on a nitrocellulose membrane and

1212 probed with MirA-His6 (or no protein as a control) and incubated with monoclonal anti-

1213 His antibody. Images of SDS-PAGE gels stained with 2,2,2 trichloroethanol (TCE) are

1214 shown as loading controls. (C) Bacterial Adenylate Cyclase Two-Hybrid (BACTH) assay

1215 of MirA and Rem. MirA and Rem as well as the Rem receiver and DNA-binding domains

1216 were fused to the T25 and T18 domains of E. coli adenylate cyclase for BACTH and

1217 assayed for lacZ activity when co-expressed in E. coli by plating $2 \mu \mathrm{L}$ on LB agar with X-

1218 gal $(160 \mu \mathrm{g} / \mathrm{mL})$ and IPTG $(500 \mu \mathrm{M})$ following a $24 \mathrm{~h}$ incubation.

1219

1220

1221 incubated 15 min followed by addition of $5 \mathrm{nM} P_{\text {motA }}$ DNA for a 15 min incubation all at RT. Reactions were separated on an $8 \%$ acrylamide gel and the DNA was stained with ethidium bromide ( $1 \mu \mathrm{g} / \mathrm{mL}) 15$ min followed by imaging with UV by BioRad ChemiDoc.

(B) Reactions were performed as in (A) but Rem was pre-incubated with $5 \mathrm{nM} P_{\text {motA }}$ DNA for 15 min prior, followed by the addition of MirA-His6.

1229 tumefaciens mirA $_{A t}$ or S. meliloti mirA ${ }_{S m}$. Colonies were inoculated into the center of 
$1230 \quad$ 0.3\% Bacto Agar (ATGN agar for A. tumefaciens and Bromfield agar for S. meliloti) and 1231 incubated $24 \mathrm{~h}$ in a humid chamber at RT. Error bars represent standard deviation for at

1232 at least 3 replicates (B) BACTH assay of MirA and Rem from S. meliloti. The mirAsm

1233 and remsm coding sequences from S. meliloti strain 1021 were fused to the T25 and

1234 T18 domains of $E$. coli adenylate cyclase for BACTH and co-expressed in E. coli.

1235 Resulting lacZ activity was evaluated by plating $2 \mu \mathrm{L}$ LB agar with $\mathrm{X}$ gal $(80 \mu \mathrm{g} / \mathrm{mL})$ and 1236 IPTG $(500 \mu \mathrm{M})$ following a $24 \mathrm{~h}$ incubation.

Figure 8. Model of MirA-dependent motility inhibition. (A) At neutral $\mathrm{pH}, \mathrm{VisN}$ and

1239 VisR activate rem expression (Rotter et al., 2006, Xu et al., 2013). Rem directly

1240 activates expression of the class II motility genes, and flagella are expressed. ExoR

1241 inhibits activity of the ChvG-Chvl system (Wu et al., 2012) (B) In acidic pH, levels of

1242 ExoR protein decrease, de-repressing ChvG-Chvi protein, which leads to activation of 1243 mirA expression. MirA forms a complex with Rem, preventing it from binding to target 1244 promoters and thereby blocking motility gene expression. 

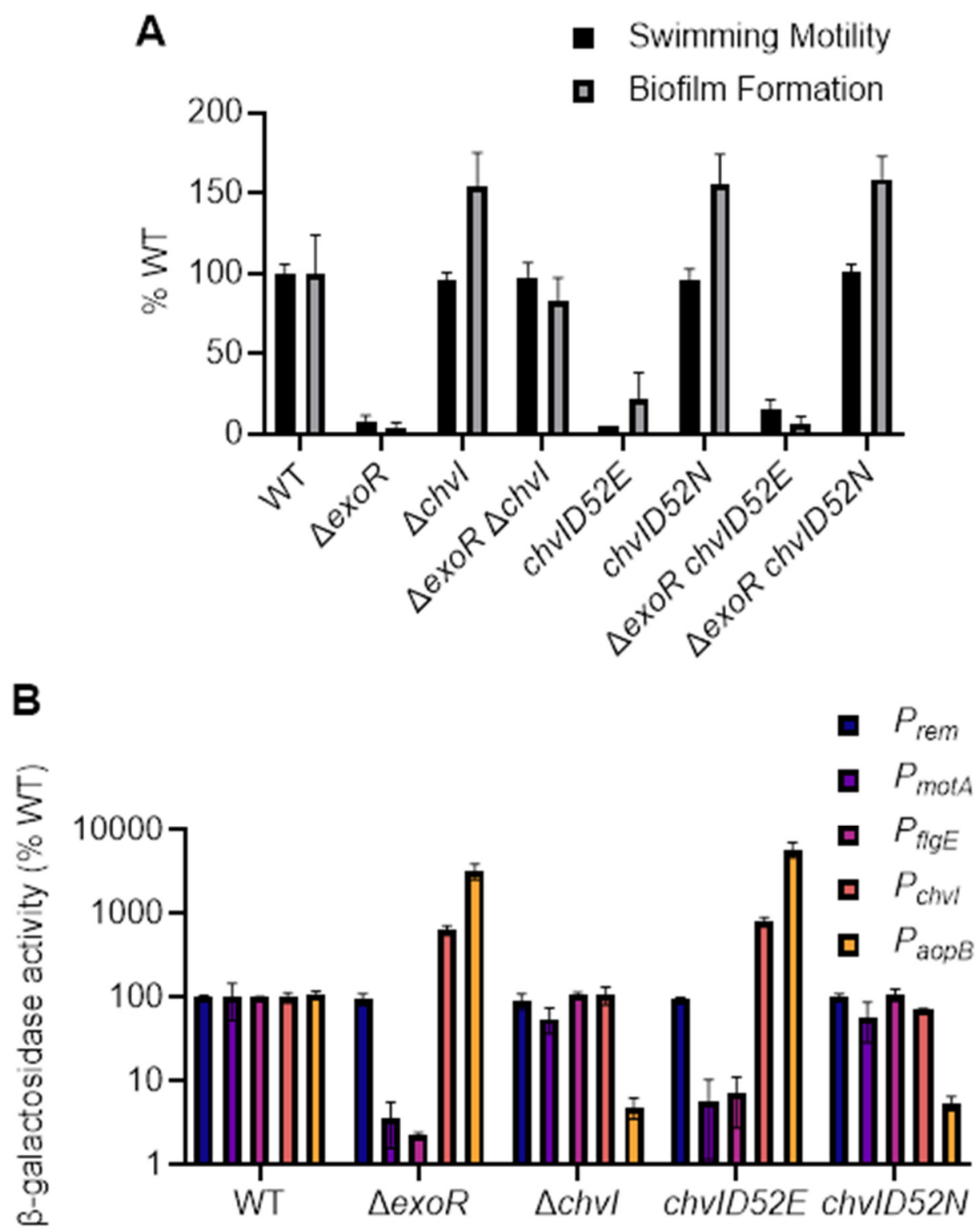
A

$\mathrm{His}_{6}-\mathrm{Chvl}^{\mathrm{D} 52 \mathrm{E}}$ :
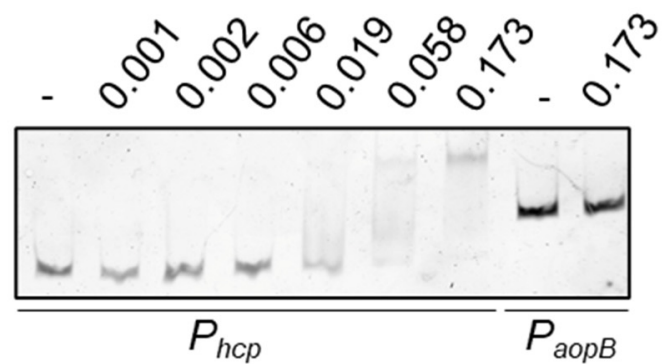

B

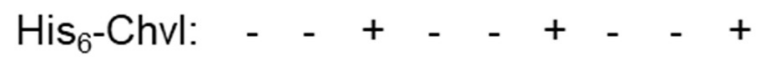

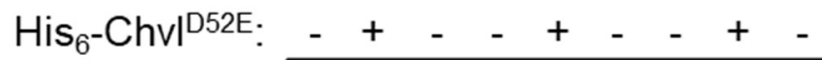

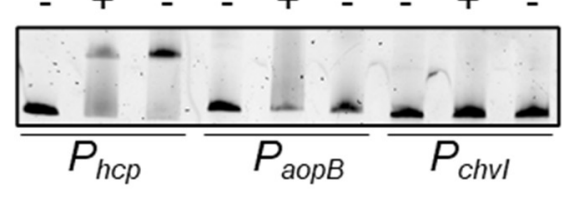

C

$\operatorname{Rem}(\mu \mathrm{M}): \quad-\quad-\quad 0.20 .41 \quad 2 \quad 3 \quad-\quad 3$

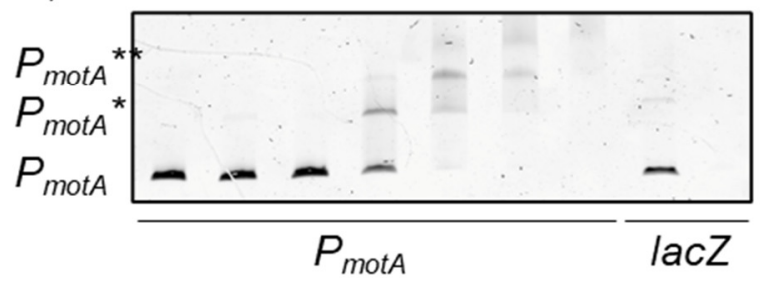

D

$\operatorname{Rem}(0.8 \mu \mathrm{M})$

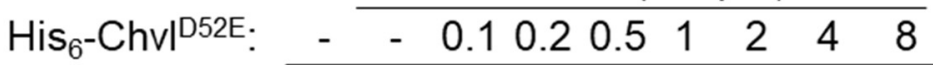

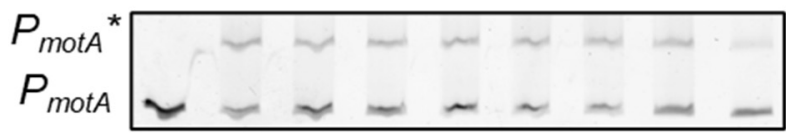




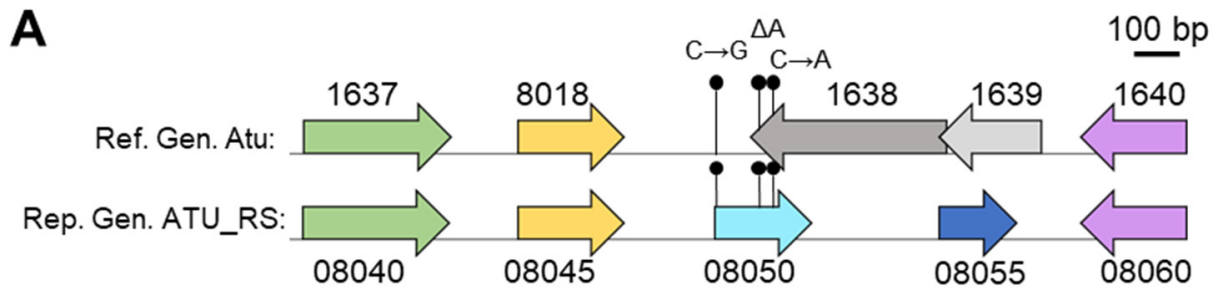

\begin{tabular}{|c|c|c|c|}
\hline Strain & Position & Mutation & AA change \\
\hline $\mathrm{BCH} 132$ & $1,625,562$ & $\mathrm{C} \rightarrow \mathrm{G}$ & $\mathrm{A} 3 \mathrm{G}$ \\
\hline $\mathrm{BCH} 133$ & $1,625,665$ & $\triangle \mathrm{A}$ & $\mathrm{FS}$ \\
\hline $\mathrm{BCH} 134$ & $1,625,700$ & $\mathrm{C} \rightarrow \mathrm{A}$ & $\mathrm{A} 49 \mathrm{D}$ \\
\hline
\end{tabular}

B

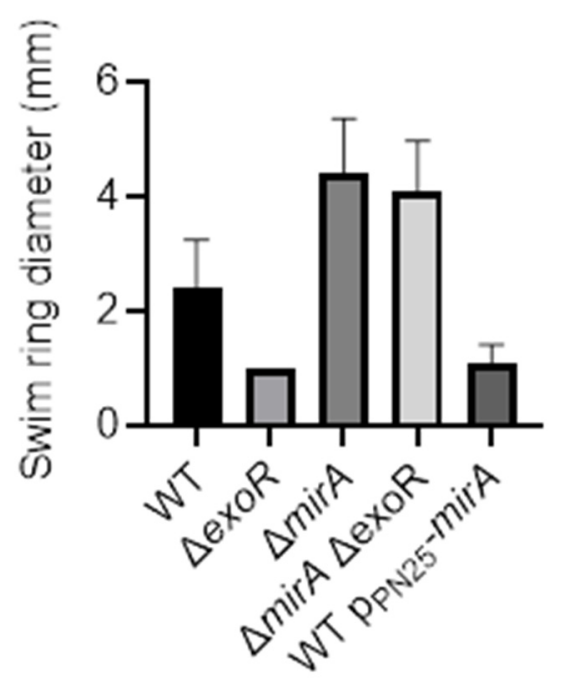

C

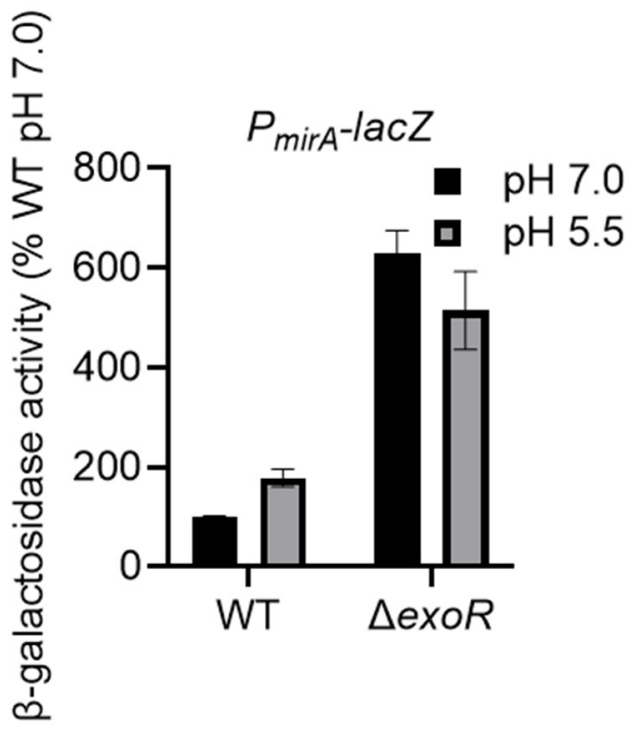

D

\begin{tabular}{|c|c|c|}
\hline & \multicolumn{2}{|c|}{ mirA-FLAG ${ }_{3}$ strain } \\
\hline & WT & $\Delta$ exoR \\
\hline$=\mathrm{LAG}_{3}$ & & \\
\hline CE & & \\
\hline
\end{tabular}

\section{Alakavuklar et al. FIG 3}


bioRxiv preprint doi: https://doi.org/10.1101/2021.06.24.449765; this version posted June 24, 2021. The copyright holder for this preprint (which was not certified by peer review) is the author/funder, who has granted bioRxiv a license to display the preprint in perpetuity. It is made available under aCC-BY-NC 4.0 International license.

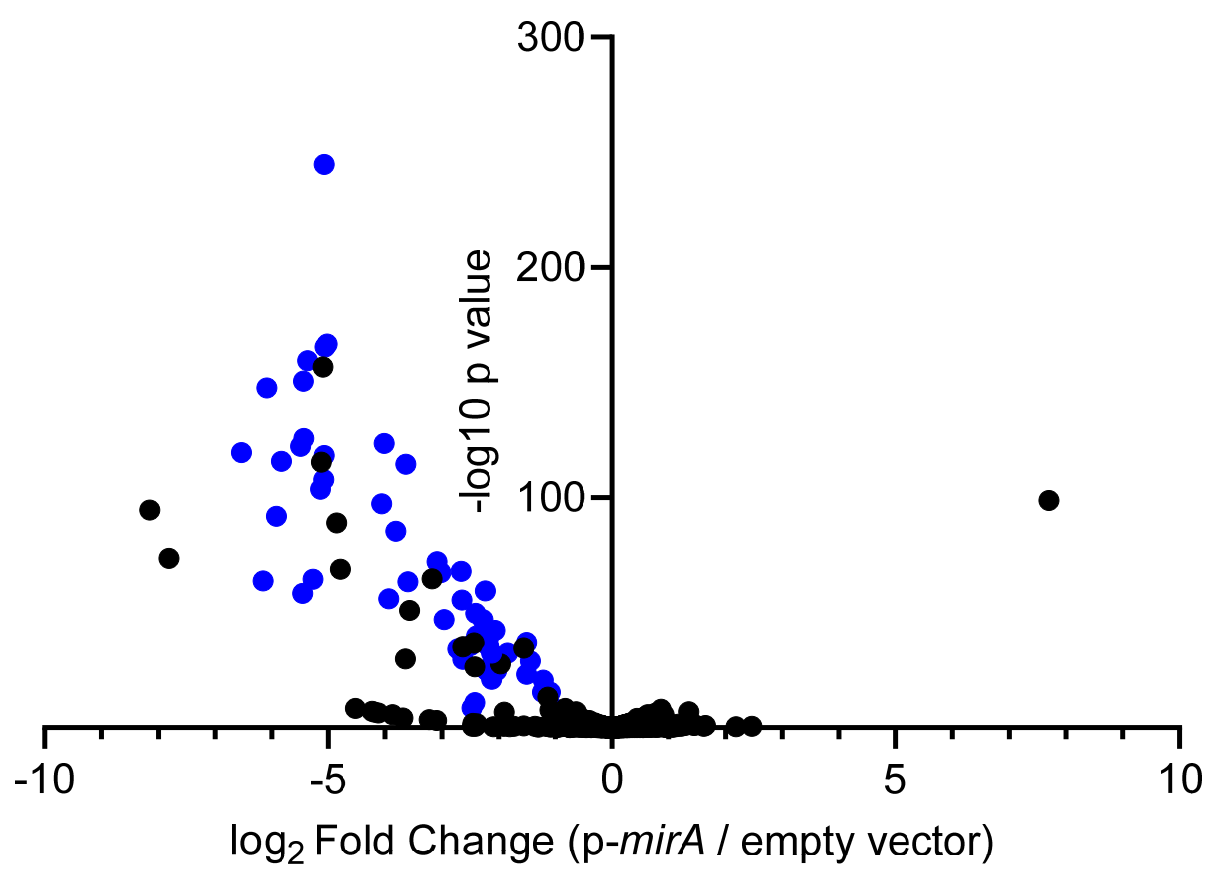

Alakavuklar et al. FIG 4 
A

$\begin{array}{cc}\text { Input } & \text { Elution } \\ & \text { Rem } \\ + & +\end{array}$

\begin{tabular}{|c|c|c|c|c|}
\hline Rem & $\begin{array}{c}\text { MirA- } \\
\text { His }_{6}\end{array}$ & Rem & $\begin{array}{l}\text { MirA- } \\
\text { His }_{6}\end{array}$ & \\
\hline $2=$ & 7 & & 5 & Rem \\
\hline & & & & MirA-His $_{6}$ \\
\hline
\end{tabular}

B

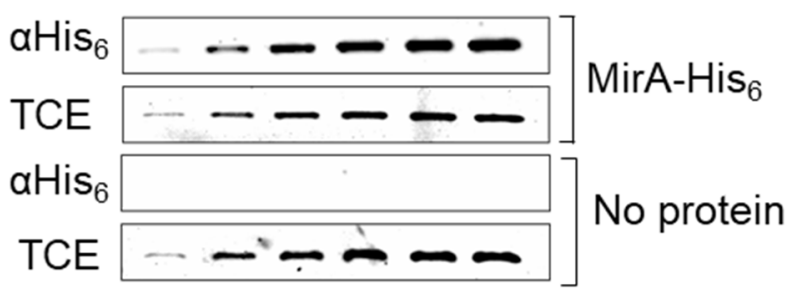

C

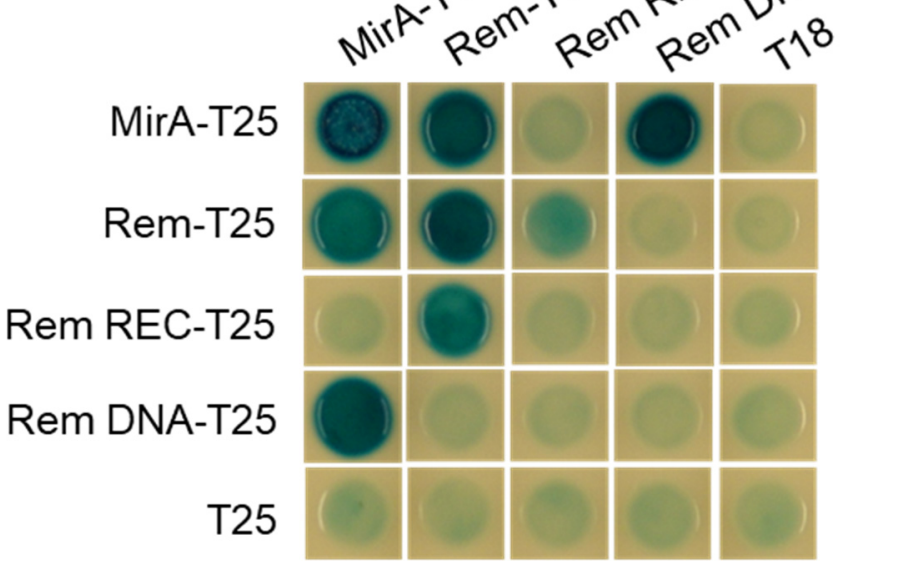


bioRxiv preprint doi: https://doi.org/10.1101/2021.06.24.449765; this version posted June 24, 2021. The copyright holder for this preprint (which was not certified by peer review) is the author/funder, who has granted bioRxiv a license to display the preprint in perpetuity. It is made available under aCC-BY-NC 4.0 International license.

A

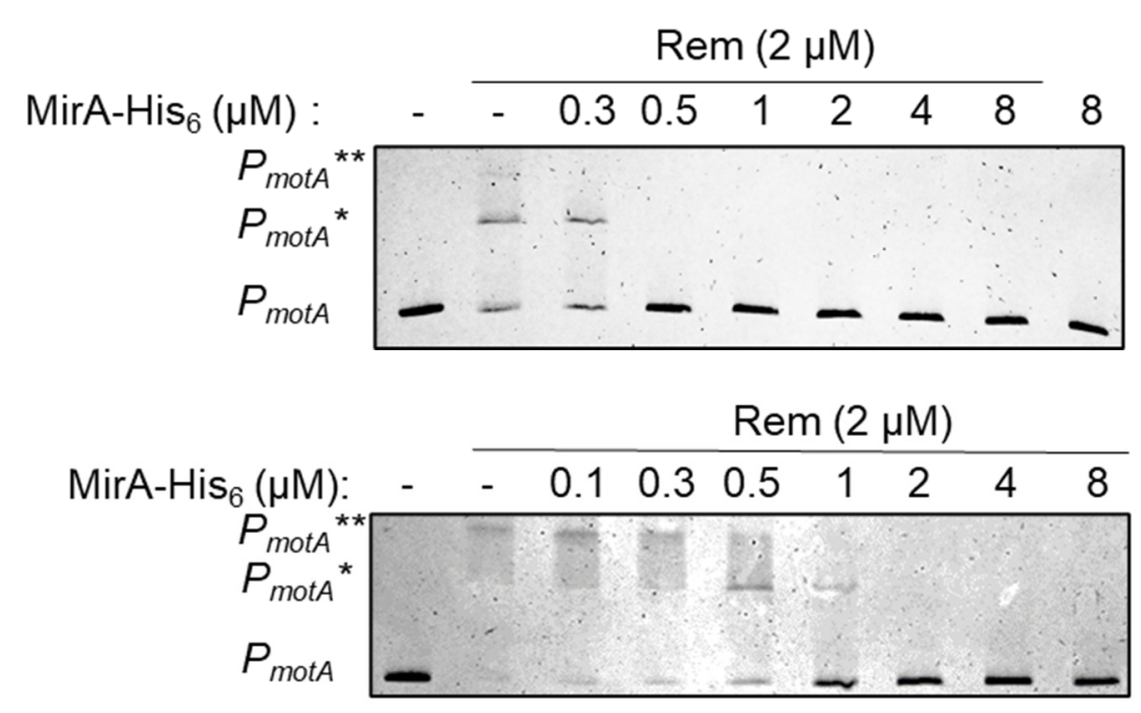

Alakavuklar et al. FIG 6 
A

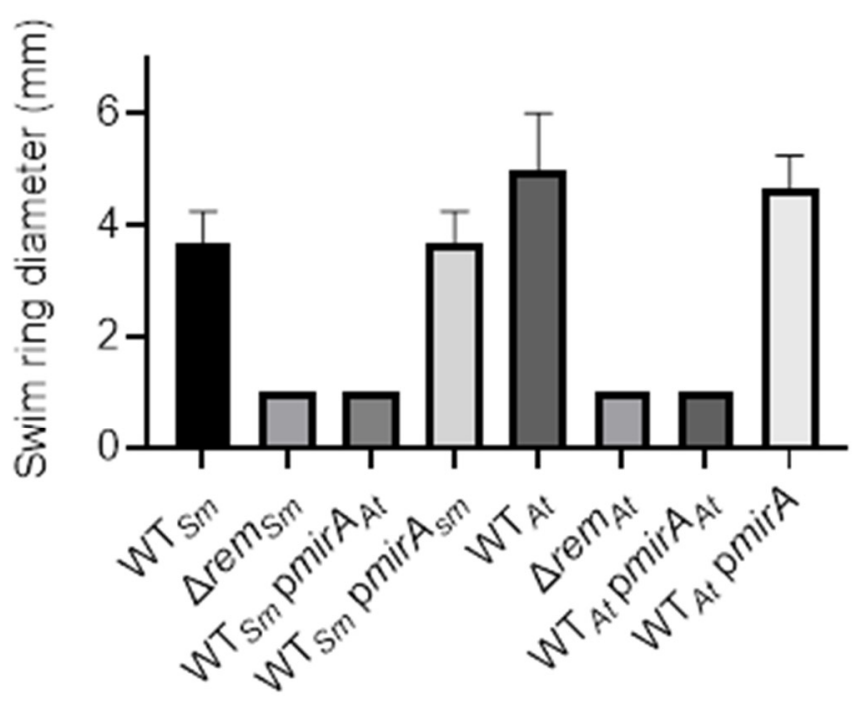

B

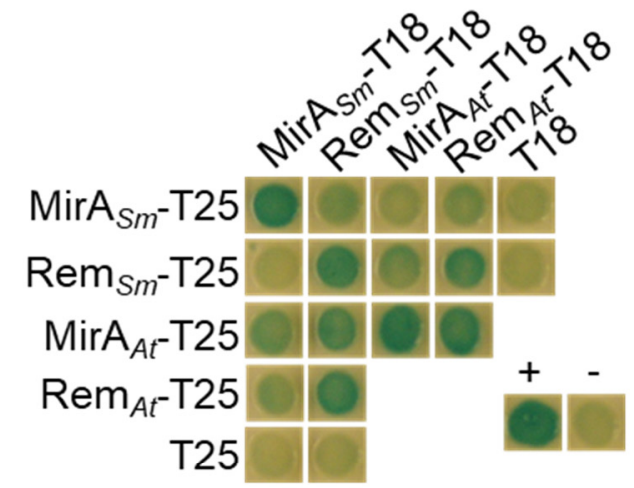


A

B

Outer Membrane

Inner Membrane

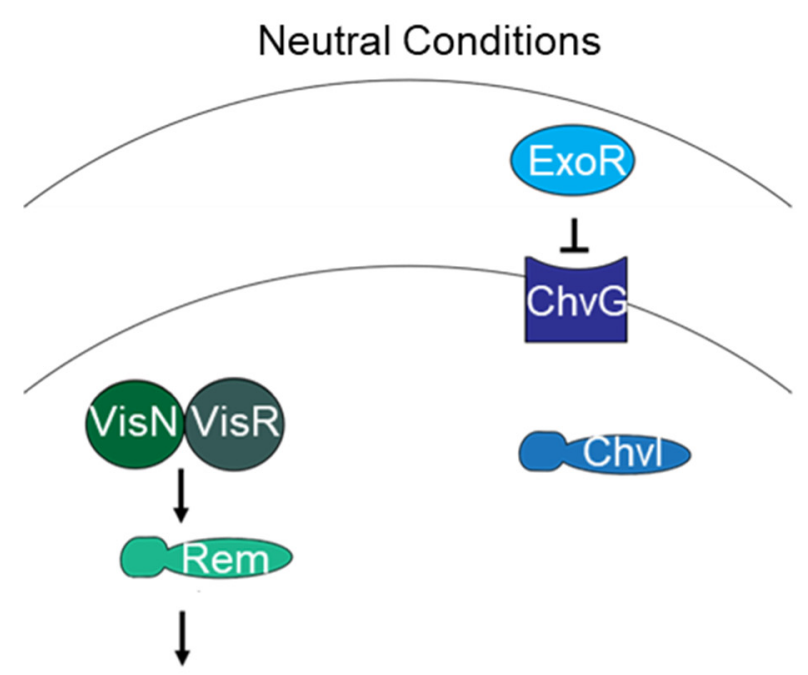

Flagellar gene

expression

Acid Stress Conditions
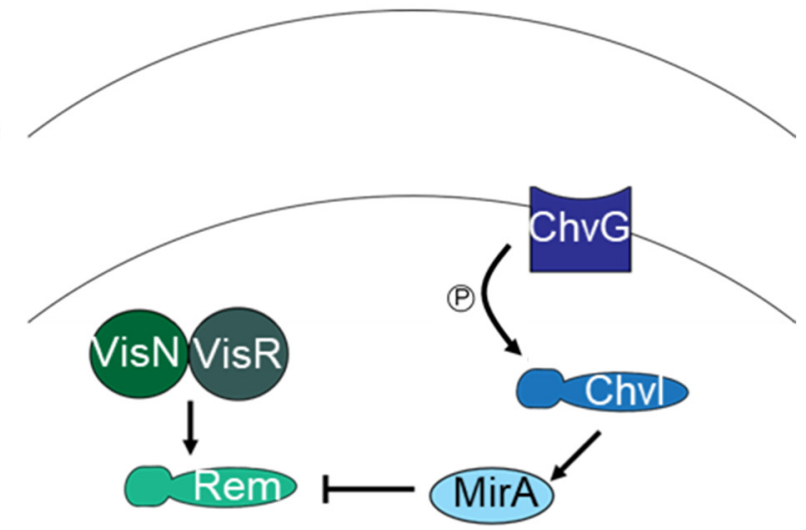

\section{Alakavuklar et al. FIG 8}

